\title{
Experimental investigation on Macroscopic Domain Formation and Evolution in Polycrystalline NiTi Microtubing under Mechanical Force
}

\author{
P. Feng and Q. P. Sun * \\ Department of Mechanical Engineering, \\ The Hong Kong University of Science and Technology, \\ Clear Water Bay, Kowloon, Hong Kong SAR, China
}

\begin{abstract}
This paper reports the experimental results on macroscopic deformation instability and domain morphology evolution during stress-induced austenite $\rightarrow$ martensite $(\mathrm{A} \rightarrow \mathrm{M})$ phase transformation in superelastic NiTi polycrystalline shape memory alloy microtubes. High-speed data and image acquisition techniques were used to investigate the dynamic and quasi-static events which took place in a displacement controlled quasi-static tensile loading/unloading process of the tube. These events include dynamic formation, self-merging, topology transition, convoluted front motion and front instability of a macroscopic deformation domain. The reported phenomena brought up several fundamental issues regarding the roles of macroscopic domain wall energy and kinetics as well as their interplay with the bulk strain energy of the tube in the observed morphology instability and pattern evolution under a mechanical force. These issues are believed to be essential elements in the theoretical modeling of macroscopic deformation patterns in polycrystals and needs systematic examination in the future.
\end{abstract}

Keywords: NiTi polycrystal, superelastic shape memory alloy, phase transformation, deformation domain and pattern evolution, macroscopic domain wall energy.

* Author to whom correspondence should be addressed; electronic mail: meqpsun@ust.hk, Tel: (852)2358-8655, Fax: (852) 2358-1543 


\section{Introduction}

Considerable effort and advances, both theoretical and experimental, have been witnessed in the past decades on the investigation of mechanical behaviors of NiTi shape memory alloys. However, in the aspect of deformation instability and the resulted domain pattern evolution of the material during phase transition, for both single crystals and polycrystals, more experimental efforts are clearly needed. Much of the previous experimental research on deformation instability in polycrystalline NiTi during phase transition (see Miyazaki, et al., 1981; Leo, et al., 1993; Shaw and Kyriakides, 1995, 1997; Brinson, 2004 and the references therein) were focused on strip and wire samples under isothermal and/or non-isothermal phase transitions. It is known that the phase transition in NiTi polycrystals is first order in nature and involves intrinsic instability and domain evolution at microscopic level. Thus complicated microstructure interaction and evolution inside the polycrystal are inherent in the transformation process. Under certain conditions, they may even percolate up to the macroscopic level, leading to macroscopic scale domain formation and evolution, as demonstrated through mechanical response and surface morphology. However, exact and detailed observations on pattern evolution in structures like thin walled tubes have not been available in the literature.

Experimental research on deformation instability of $\mathrm{NiTi}$ in microtube 
configuration started only in recent years (Berg, 1997; Li and Sun, 2000, Sun and Li, 2002; Li and Sun, 2002). It is motivated by the successful application of microtubes in human implants and surgery instruments. In addition to the facilitation of biaxial loading, the advantage of using the tube configuration is that an isolated high strain domain (martensite phase) can survive in the tube after its nucleation from the austenite matrix and that deformation patterns associated with the domain evolution during the loading process can be produced and observed. The microtubes are usually polycrystalline and nano-grained, with an outer diameter ranging from the smallest of 0.06 to several millimetres and length of a few meters. They are manufactured by cold drawing, therefore the texture of the tubes could be very strong which will give significant anisotropy in both elastic and transformation properties. Previous preliminary study by the authors showed that the transformation process in such a nano-grained polycrystal involves both micro- and macroscopic instabilities and is multi-scale in nature (see Bhattacharya, 2003; Bhattacharya, et al., 2004 and the references therein) and that macroscopic deformation instability under tension manifested itself not only through sharp stress jumps in the nominal stress-strain curves of the tube but also through the various evolving deformation patterns as revealed by careful tube surface observations.

This paper reports some of the key results from the authors' recent experimental 
investigation on macroscopic domain formation and evolution in the superelastic polycrystalline NiTi microtubing under uniaxial tensile force. The purpose of the research is to obtain quantitative information on the spatiotemporal evolution of the deformation patterns through systematic measurement and eventually to provide critical data for future theoretical development of this type of material. The material properties and test procedures are described in section 2. Section 3 gives a detailed description of the experimental results. Discussions of the results are given in section 4 and the conclusions are given in section 5 .

\section{Materials and Experimental Procedures}

2.1. Material characterization and sample preparation

The material used in the experiment was a commercial polycrystalline NiTi tube with composition of 55.4 wt.\% Ni (Nitinol Devices \& Components, USA). The outer and inner diameters of the tube were $1.78 \mathrm{~mm}$ and $1.47 \mathrm{~mm}$ respectively. The grain size was in the range of 50 100 nm. Using differential scanning calorimeter (DSC), the measured austenite finish temperature $\left(A_{f}\right)$ of the tube was $1{ }^{\circ} \mathrm{C}$ so the material is in the austenite state at room temperature $\left(23^{\circ} \mathrm{C}\right)$ and will exhibit superelastic behavior under stress. The sample was chemically etched by hydrofluoric acid into a dog-bone shape and then mechanically polished by fine grained sand papers to reach a final surface roughness of $0.15 \mu \mathrm{m}$. The dog-bone shape sample is sketched in Figure 
1. The dimensions of the sample used in the experiment of this paper are: the outer diameter $D=1.73 \mathrm{~mm}$, the gauge length $L=40 \mathrm{~mm}$, and the total length $L_{0}=76 \mathrm{~mm}$.

\subsection{Mini testing system for microtubing}

An electro-mechanical-optical testing system suitable for NiTi microtubing was developed to facilitate a series of precise loading and measurements such as high resolution displacement controlled loading, high speed data acquisition and synchronized recording of the load-displacement response and image of the tube surface. The testing system consists of four subsystems: loading, measurement, surface observation and automation (see Fig. A1 in Appendix A). The loading subsystem was designed to apply a displacement-controlled loading to the tube with elongation rate ranging from $0.1 \mu \mathrm{m} / \mathrm{s}$ to $5 \mathrm{~mm} / \mathrm{s}$, suitable for both quasi-static and fast loadings. The measurement subsystem was to measure the axial elongation, rotation, axial force and torque on the specimen, from which the nominal stress and strain relations of the specimen were deduced. The observation subsystem employed an imaging camera, high speed motion camera, measuring microscope, surface profiler and infrared camera to observe and measure the relevant quantities on the specimen surface. The automation subsystem is for automatic testing, including control of the loading process, high-speed data acquisition, image acquisition, real-time monitoring and synchronizing of the measured data and images. A detailed 
description of each subsystem can be found in the work of Feng (2005). The four subsystems were assembled and tested with a standard specimen. The stress-strain curves obtained by the developed systems were compared with those by a commercial universal testing machine so that the reliability and accuracy of the systems were assured. The system is able to perform tension-torsion combined loading, cooling and heating. In this paper it was only used for axial loading of tube at room temperature $\left(23^{\circ} \mathrm{C}\right)$.

\subsection{Experimental setup}

The austenite to martensite $(\mathrm{A} \rightarrow \mathrm{M})$ phase transition of the tube was induced by a quasi-static displacement-controlled uniaxial tension at room temperature on the manufactured testing system (see Fig. A1 in Appendix A). In order to minimize the self-heating effect caused by the transformation latent heat, all tests were performed at a very low elongation rate of $1.2 \mu \mathrm{m} / \mathrm{s}$ which gave a nominal strain rate of $3 \times 10^{-5} \mathrm{~s}^{-1}$. The loading frame was precisely manufactured and the axis of the tube and the loading direction were carefully aligned to achieve a negligible bending moment caused by off-axis tension. The special tubing grips transferred the tensile force to a uniformly distributed tube surface shear stress at the two end portions of the tube, which effectively avoided stress concentration and produced a uniform tensile stress in the tube cross section over the $40 \mathrm{~mm}$ middle portion (gauge section) of the tube. 
Deformation domain morphology of the tube surface during loading were obtained by different apparatus such as imaging camera, surface profiler, microscope and high speed camera so that comparison between the measured results were possible. An infra-red camera was used to record the tube surface temperature evolution. The control of the loading process, data acquisition and the synchronized display of the stress-strain curves and surface morphology on screen, etc., were all facilitated by customized software.

\section{Experimental Results}

The experimental observations reported in this section were repeatable in the tests of the same specimen for more than fifty loading-unloading cycles with time intervals ranging from several hours to several weeks, though the cyclic effect made the stress drop value smaller and domain fronts less clear. The observed deformation instability and domain evolution were also repeatable in the tests on other specimens whose gauge length ranges from $30 \mathrm{~mm}$ to $110 \mathrm{~mm}$. Even for the specimens directly cut from the as-received tube with a uniform cross section, similar phenomena were observed.

We will first outline the main deformation features of the tube during tensile loading/unloading in section 3.1. A detailed description of the deformation and domain morphology evolution during forward $(A \rightarrow M)$ and reverse $(M \rightarrow A)$ 
transformations will be given in sections 3.2-3.6. Section 3.7 describes the path dependence of domain morphology.

\subsection{Main deformation features of NiTi tube during quasi-static loading/unloading}

The typical measured macroscopic nominal stress-nominal strain (S-S) curve of the tube during loading and unloading is shown in Fig. 2(a) (throughout this paper, the nominal stress is obtained by dividing the measured force by the initial cross-section area of the gauge section, the nominal strain is the extensometer elongation divided by the gauge length which is $25 \mathrm{~mm}$ ). In the test shown by the curve, the sample ( $\mathrm{L}=40 \mathrm{~mm}$ ) was elongated or shrunk at a constant rate of $1.2 \mu \mathrm{m} / \mathrm{s}$ and the total test time was 2001.5 seconds.

During the loading process, the deformation consists of the following stages which can be identified from both the S-S curve and the surface morphology in Fig. 2. They are:

1. Linear elastic deformation of austenite followed by macroscopically homogeneous but nonlinear deformation until the peak load. The later corresponds to small amount transformation of the austenite polycrystal (pre-burst incubation), see "Loading I" in Fig. 2(a) and the corresponding photos of surface in Fig. 2(b) $A \sim B$; 
2. Dynamic formation (starting at the peak load) of a macroscopic deformation domain and its rapid growth into a helical shaped band, see $B \sim C$ in Fig. 2(a) with the magnified S-S curve. The corresponding surface morphology of the tube is shown in $B \sim C$ of Fig. 2(b). The variation of the domain wall velocities (at the tip and the middle point of the wall) with the applied nominal stress during this rapid growth are given in section 3.2;

3. Stable quasistatic helical domain growth (Fig. 2(a) $C \sim E$ ) until the domain self-merging at which the domain topology changed from a simply connected domain to a multiply connected one (here a cylindrical domain, see Fig. 2(a) $E \sim F$ with the enlarged S-S curve during merging). The tube surface morphologies are shown in Fig. 2(b) $C \sim F$ ). At the same time the fronts at the two ends of the cylindrical domain branched into several sharp tips, see Fig. 2(b) $E \sim F$ and section 3.3 for details;

4. Further growth of the cylindrical domain via the convoluted propagation of the macroscopic branched domain fronts, exhibiting a nearly steady state advancing and rotating pattern in the tube, see "Loading-IV" in Fig. 2(a) and Fig. 2(b) $F \sim G$. The details are given in section 3.4.

To avoid the end effect at the shoulders (non-uniform cross-section) of the gauge section, the specimen was only loaded until about 30\% of the gauge length section 
was transformed (the cylindrical domain was about $12 \mathrm{~mm}$ long) and then was unloaded. The unloading process involved the following deformation stages which were not simple reversals of those in the loading process and the reverse transition took place on a much lower stress plateau due to hysteresis of transformation. They are:

1. Elastic unloading with the tube surface pattern almost unchanged, see Fig. 2(a) $G \sim H$ and Fig. 2(b) $G \sim H$;

2. On the lower stress plateau, shrinkage of the cylindrical domain took place through the reverse convoluted motion of the branched front, see Fig. 2(a) $I \sim K$ and Fig. 2(b) $I \sim K$;

3. Front instability happened when the length of the cylindrical domain is reduced to a certain value, i.e., the original branched front switched to a smooth one and the domain shape became an inclined ring (see $K \sim L$ in Fig. 2(a) and 2(b)). (Note: Reloading the tube to the upper stress plateau gave the opposite result, i.e., the smooth front of the inclined ring switched back to the serrated shape with several long sharp tips, see section 3.7 for details);

4. Further unloading changed the smooth ring topology, via the merge of the two fronts, into an lenticular inclusion which was soon annihilated dynamically, signalling the completion of the reverse transformation process, see Fig. 2(a) 
“Unloading-II” and the enlarged S-S curve, Fig. 2(b) $L \sim M$ and section 3.6 for details;

5. Elastic unloading of the austenite (Unloading-I in Fig. 2(a)).

The above key features of deformation are summarized in Table 1. A total of seven movies on the deformation processes are available at the website: http://www-mech.ust.hk/ meqpsun/.

3.2. Pre-burst incubation and dynamic domain formation (Stages Loading-I and II)

The observation of this stage is realized by recording the force-displacement response (or nominal S-S curve) and the tube front-surface and back-surface morphologies synchronistically. The morphologies was recorded by a CCD camera (TK-C1381EG, JVC, Japan) at a rate of 25 frames per second (fps) and by a high speed camera (Motion Scope PCI, Redlake, USA) at a rate of 250 fps respectively, both cameras were equipped with a telescopic lens (Zoom 7000, Navitar, USA). Deformation at this stage could be divided into three sub-stages: (i) homogeneous elastic deformation of austenite; (ii) macroscopically homogeneous and stable transformation from austenite to martensite, referred to as "pre-burst transformation" or "incubation" (Fig. 3); and (iii) dynamic domain formation process which started at the peak load and was accompanied by a rapid load decrease in the S-S curve, as shown in Fig. 4. 
At sub-stage (i), the Young's modulus of the polycrystal in its austenite phase (A phase) was measured from the linear part of the stress-strain curve as $35 \mathrm{GPa}$. When the stress level was over $305 \mathrm{MPa}$ (the corresponding strain value is $0.93 \%$ ), deformation entered sub-stage (ii) where the gradual bending of the S-S curve with increasing deviation from linearity represented the $\mathrm{A} \rightarrow \mathrm{M}$ phase transition. To show that such nonlinearity is indeed due to phase transition, the loading was stopped before the peak and then the specimen was unloaded. A small S-S loops were observed demonstrating that small amounts of phase transition had happened (as shown in Fig. 3, also see Li and Sun, 2002) and was responsible for the nonlinearity. In this sub-stage the tangent modulus of the polycrystal was decreased to as small as 6 GPa at point $B$ and finally approached even smaller values at the peak load point $a$ in Fig. 4(a).

At sub-stage (iii), soon after the stress reached the peak value at point $a$ (about $383 \mathrm{MPa}$ ), the deformation became inhomogeneous and uncontrollable through the dynamic formation (in the sense that it occurred very rapidly, see the time coordinates in Fig. 4(a)) of a macroscopic deformation domain with a concomitant rapid nominal stress decrease (from $383 \mathrm{MPa}$ to $367 \mathrm{MPa}$ ) as shown in the sequences $a \sim n$ in Fig. 4(a). The domain is actually the most transformed high strain region (martensite) of the tube and was separated from the almost untransformed austenite (matrix) by a 
distinct domain boundary (or front region) across which the strain changed rapidly. The domain could originate at any location of the measurement section depending on the defects of the material (here it was at the middle portion of the tube). Figure 4(b) shows the corresponding tube surface images chosen from a sequence of photos taken at $0.08 \mathrm{~s}$ and $1.56 \mathrm{~s}$ time intervals respectively. The whole time period during which the load decreased can be clearly divided into two stages. The first stage lasted only about $0.48 \mathrm{~s}$ during which the load decreased very rapidly (points $a \sim g$ ). In the second stage (which lasted about $7.80 \mathrm{~s}$ ) the load decreased much more slowly (see points $g \sim l)$. After this the load reached a stable plateau value and the corresponding domain became a helical band with two spike-like tips. The aspect ratio (length to width) of the band was about 14 as shown in $n$ of Fig. 4(b) (also see Fig. 7(a)).

To increase the time resolution for the very short period of initial domain formation (see Fig. $4 a \sim d$ ), a high speed camera was used to record the image of the tube surface at a rate of $250 \mathrm{fps}$ and the photos are shown in Fig. 5. It can be seen that the initial domain was oriented at an inclination angle of about $60^{\circ}$ to the loading axis. The evolution of the initial shape and length of the domain was difficult to identify because the domain emerged very quickly from a homogeneously deformed tube and the domain boundary was quite diffusive. However, the domain width could be estimated as about $0.12 \mathrm{~mm}$ from the figure with relative ease. This value is in the 
same order of the wall thickness of the tube $(\approx 0.13 \mathrm{~mm})$. The subsequent growth process of this newly formed domain is shown in a series of photos in Fig. $4 d \sim n$. It can be seen that the initial inclined narrow domain quickly evolved into a lenticular shaped domain that spiraled around the tube at an angle of $60^{\circ}$ to the load axis. By surface profiling the width (W) of the transition profile from austenite to martensite on the tube surface was in the range of $0.1 \sim 0.2 \mathrm{~mm}$ as shown in Fig. 6. It must be pointed out that this value is proportional to the thickness of the tube wall and therefore is not an objective measure of the real front thickness which will be discussed in section 4.2.

The data from the synchronized measurements (acquired at $25 \mathrm{fps}$ ) were used to determine the front velocity at the helical domain tip (denoted by $V_{t}$ ) and the front normal velocity at the mid-point of the long front $\left(V_{m}\right)$. The variations of $V_{t}$ and $V_{m}$ with time, nominal strain and stress drop are plotted in Fig. 7. It can be seen that the tip velocity $V_{t}$ (the maximum is about $40 \mathrm{~mm} / \mathrm{s}$ ) was much higher than the mid-point velocity $V_{m}$ (the maximum is about $5 \mathrm{~mm} / \mathrm{s}$ ), giving a much faster domain lengthening than its lateral thickening. Both velocities decreased rapidly with time and with the decrease in stress (driving force). With the front velocity data and the width of front profile $(\delta)$, the local axial strain rate of a surface material element at the middle point of the long front (when it is swept by the front) can be estimated as (assuming total 
strain $\varepsilon$ varies linearly over $\delta) \quad d(\varepsilon) / d t=\varepsilon^{T} V_{m} /\left(\delta \cos 30^{\circ}\right)$. Here $\varepsilon^{T}$ is the transformation strain (of the order of $5 \%$ ), $\delta$ is of the order of $0.1 \mathrm{~mm}$. It can be seen that under the nominal quasi-static strain rate of $3 \times 10^{-5} \mathrm{~s}^{-1}$ the local strain rate of the material can reach $10^{0} \sim 10^{1} \mathrm{~s}^{-1}, 5$ to 6 orders of magnitude higher than the nominal strain rate due to the dynamic event. The local strain rate at the tip region of the domain could be even higher.

Remark 1. The values of front velocity above are conservative since they depend on the image acquisition rate (unit: fps). Even higher front velocity can be expected with higher time resolution. For example, using 250 fps rate by a high speed camera, we estimated a middle front velocity as high as $2000 \mathrm{~mm} / \mathrm{s}$.

Remark 2. The temperature effect of latent heat was checked for both dynamic and quasi-static processes by using an infrared camera. The measured local temperature increase during the dynamic growth was less than $2{ }^{\circ} \mathrm{C}$ (note: this is not very accurate since the frame rate of the camera is only $25 \mathrm{fps})$. At the late stage of domain growth on the plateau (see section 3.3) the front velocity was only about $0.06 \mathrm{~mm} / \mathrm{s}$, in such slow motion of the front almost no temperature increment could be detected (less than $\left.0.2^{\circ} \mathrm{C}\right)$

Remark 3. It has been seen that the formation and growth of the helical domain broke the symmetry of axial loading and tube geometry. Such symmetry-breaking 
morphology evolution might be attributed to the total energy (strain energy plus domain wall/interface energy) preference of the shape of the high-strain domain in which the transformation strain is shear in nature. A detailed discussion of the domain wall energy and domain shape will be given in section 4 .

3.3. Quasi-static helical domain growth, self-merging and front branching (Stage Loading-III)

The dynamic domain formation completed as the force dropped to the plateau. The tube then consisted of a simply connected helical domain and the surrounding austenite matrix as shown by photo $n$ in Fig. 4(b). Further elongation of the tube led to the growth of this macroscopic helical domain by lengthening and thickening, which proceeded in a stable and controlled manner via visible quasi-static movement of the domain front. The corresponding load was kept roughly unchanged on the stress plateau (Fig. 2(a)).

With further loading the low-strain matrix sandwiched in the growing high-strain domain became more and more narrow. Eventually the nearest parts of the two fronts started getting touched each other, indicating the self-merging of the domain (see Fig. 8). The merge is again an unstable and dynamic process in the sense that it occurred in an uncontrollable and swift manner as shown by the load drop in the enlarged S-S curve in Fig. 8(a) with time coordinates. The corresponding tube surface images are 
shown in Fig. 8(b) (the photos) and (c) and (d) (3D and plane views respectively). If we borrow the terminology from material science and presume that front energy (interfacial energy) exists in the polycrystal studied here, the driving force for such a dynamic merge could be from the reduction of the system's total front energy, which caused the two closely paralleled fronts to "attract" each other and led to the speeding-up of the merging process. At the same time, the merge disconnected the original narrow matrix region in the middle portion of the domain into two regions (left and right portions with long tips in a beak-to-beak manner as shown in Fig. 8(d)). The process that followed the merge was a gradual stable retreat of the two tips of the austenite phase back to the left and right matrix (see Fig. 8(c) and (d)). Thus the helical domain turned into a cylinder-like one.

Remark 1. The self-merging process changed the domain topology from the original simply connected helix to a multiply connected cylinder. Topological transitions commonly existed in the transformation process of materials such as particle merging during the coarsening (e.g. Leo et al., 1998). The deformation domain of the tube would experience several topology transitions in the loading and unloading process as we will see in the following sections.

Remark 2. It was also observed in the present experiment that if the sample were unloaded prior to the domain self-merging, the domain pattern would evolve 
reversibly once the load was reduced to reach the lower force plateau. The retreat of the domain front caused the helical domain to shrink almost along its original path and eventually the domain vanished dynamically (we will be back to this issue in section 3.7).

Remark 3. The fronts at the two ends of the cylindrical domain were not smooth (even a macroscopic smooth straight front was very rough microscopically). Instead, they were macroscopically branched with several tips. The formation of the branched shape during self-merging is still under investigation. The in-situ movie of the tube surface morphology clearly showed that branching took place even before the merge started (see photos $a \sim d$ in Fig. 8(b)). One simple presumption in explaining the branching is that it helps to reduce the strain energy of the domain (especially near the otherwise smooth front) at the expense of increasing the total front length and therefore the total interfacial energy of the front. As shown in Fig. 9, the reduction of strain energy is realized by making the angles $\theta_{1}\left(\approx 72^{\circ}\right)$ and $\theta_{2}\left(\approx 63^{\circ}\right)$ of the two inclined planar fronts as close as possible to the angle $\theta_{0}$ of the "invariant-line" or the "kinematical compatibility" (Bhattacharya, 2003). The value $\theta_{0}=58.4^{\circ}$ is calculated as shown in Fig. 10, using Mohr's circle method, from the measured transformation strain components $\varepsilon_{x}^{T}$ and $\varepsilon_{y}^{T}$ which are obtained by the method called in-situ profilometry (Feng and Sun, 2006). It is noticed that the mean contour 
of the zigzag front is still being kept roughly perpendicular to the loading axis so as to reduce the bending and torsion of the tube caused by a cylindrical domain with an otherwise inclined interface.

3.4 Steady-state cylindrical domain growth via convoluted motion of the front (Stage Loading-IV)

In this stage of deformation, the axial force remained on the plateau of the S-S curve ( $F \sim G$ in Fig. 2(a)) and the corresponding tube surface morphologies are shown in Figs. 2(b) and 11(a). The growth of the cylindrical domain almost reached a steady state and was realized by the convolution of its fronts. There are two important features in this growth stage. One is that all branches of the finger-like front were nearly identical to each other and oriented at the same angle with the loading axis. The second is that the normal velocities at different locations of the front were different (e. g., the branch tip moved faster than the rest of the front) under external uniform loading but were highly coordinated. It was such highly coordinated non-uniform front motion that leads to the above observed rotation/advance of the front pattern as shown in Fig. 11(a) (photos) and 11(b) (perspective contour drawing). The tube itself was straight without rotation but the image of the domain pattern, due to the convoluted motion of the front, kept changing in an advancing-and-rotating manner. The transformation strain of the domain (i.e., the average strain of the most 
transformed polycrystal, see Brinson et al., 2004) was measured by a high resolution surface profiler. As shown in Fig. 10, the total axial strain is $6.51 \%$ which agrees very well with the results by the extensometer in the present investigation.

Remark 1. During the steady-state motion of the front, the mean contour of the branched front shown in Fig. 9(d) was globally maintained perpendicular to the loading axis as shown in Fig. 11(a). Again a presumptive explanation for the branching is that it helps to reduce the strain energy stored near the front of the cylindrical domain at the expense of increasing the surface energy of the front (see Fig. 11(c)). In other words, the domain with branched front is thermodynamically more stable than the domain of the same volume but with a smooth perpendicular front. While the reason for keeping the mean contour of the two zigzag fronts globally perpendicular to the loading axis is that such a configuration could minimize the bending and torsion of the tube. For example, if the two fronts of the cylindrical domain were globally inclined (as shown in Fig. 12(a)) the bending due to the distortion of the tube would monotonically increase with the domain length.

Remark 2. It was observed that the number of branches could be different for different tests using the samples cut from the same long tube (compare the six branches in Fig. 9 with four branches in Fig. 11). This might be due to the inhomogeneity of samples and needs to be clarified in future studies. Except the 
number of branches, all other aspects of the observed phenomena for different samples are the same.

Remark 3. Up to now, the domain morphology evolution during the loading process can be summarized as the sequence of from the formation of helical domain with a smooth front to its growth and front branching, then to the self-merging and formation of a cylindrical domain by topology transition, and finally to the steady state growth of the cylinder-like domain by convoluted motion of the branched front while the mean contour of which is remained perpendicular to the loading axis. However, as will be shown below, the pattern evolution during unloading is not a simple reversal of those in the loading process but exhibits a rather strong loading history dependence.

3.5 Reverse transition during unloading — reverse convolution and switching from branched front to smooth front (Unloading-IV and III)

The unloading first led to an almost linear elastic response of the tube until the force reached the lower stress plateau where reverse transition proceeded through the reverse convolution of the front. The shrinkage of the domain was a gradual and stable process under quasi-static unloading and took place at the lower stress plateau (about $205 \mathrm{MPa}$ ). For the stage of Unloading-IV (I K of Fig. 2(a) and 2(b)) the shrinkage was basically a simple reversal of that in loading. However, at the stage of 
Unloading-III two events were observed: one is the gradual inclination of the front followed by the front switching from a branched to a smooth one at a critical domain length; the other is the breaking of smooth ring-like domain and dynamic domain vanishing which will be shown in section 3.6.

Before the branched front switched to a smooth one, the branched front of the cylindrical domain had gradually become inclined during unloading (as shown in Fig. 12(a)). When the domain length (i.e., the length along axial direction) was reduced to about $1.10 \mathrm{~mm}$, the inclined branched front became unstable and switched to a smooth one dynamically through the fast retreat of the tips at a speed of the order of $10 \mathrm{~mm} / \mathrm{s}$ (see the last two pictures in Fig. 12(a) and Fig. 12(c) of a higher time resolution). A series of schematic drawings based on our observations on the evolution of a branched front into a smooth one are shown in Fig. 12(b). When in the form of a smooth inclined ring, the domain continued to shrink for a while during further unloading with its two fronts almost remaining parallel.

Remark 1. One mechanism responsible for the gradual inclination of the original globally perpendicular front into a globally inclined one is the competition between the strain energy and the energy due to the self-bending and self-torsion of the tube. As the cylindrical domain shrank and the domain length became shorter, the self-bending energy due to the inclined front was much reduced and the strain energy 
of the domain became important and dominated the domain orientation, which made the branched front become globally inclined. Both the strain energy and surface energy of the domain would be smaller than those of a domain with the same length but with branched and globally perpendicular fronts.

Remark 2. The front switching could be explained as follows: as the axial length of the inclined cylinder-like domain became short (i.e. an inclined ring), the ratio of the surface energy over the strain energy became large so that the surface energy dominated the morphology. This made the branched front switch to a smooth one so as to reduce the surface energy.

3.6 Ring-like domain breaking and dynamic annihilation (Stage Unloading-I and II)

Further unloading led the reverse transformation process to completion through two sub-stages: the breaking of the smooth ring-like domain into a C-shaped inclusion by topology transition and dynamic annihilation of this C-shaped domain, as shown in Fig. 13.

The first sub-stage ring-like domain shrinkage was accompanied by a gradual load increase until the domain was "broken” into a C-shaped inclusion (see $b \sim f$ of Fig. 13). The deformation of this stage was stable and can be controlled. It was noticed that the front orientation varied along the ring-like domain front and the domain shrank non-uniformly. For the given elongation (unloading) rate, when the domain 
length was reduced to below $0.71 \mathrm{~mm}$ (see point $b$ ) its shrinkage was accelerated, and this caused a small but obvious stress increase (see $b \sim f$ in Fig. 13(a)). It seemed that, like the attraction in self-merging during loading, an attraction force emerged on the two fronts when the distance between the two fronts reached a certain value. It was this attractive force that caused the accelerated shrinkage. This eventually led to the topology transition changing the ring-like domain into a C-shaped inclusion with an aspect ratio of about 20 (5.4 mm length/0.26 mm width, see $f$ in Fig. 13(b) and 13(c)) (refer to the movie on the website http://www-mech.ust.hk/ meqpsun/).

In the second sub-stage the C-shaped domain further shortened and thinned with load fluctuations for a short period ( $f \sim h$ in Fig. 13(a)), then shrank dynamically by fast front retreat and eventually vanished as shown by both the very steep load rise (see $h \sim n$ in Fig. 13(a) and the surface images in 13(b) and 13(c)). After the domain annihilation, the matrix (austenite phase) deformed linearly for the rest of the unloading process and the S-S curve almost coincided with that of the loading process.

Remark 1. It was found that the magnified S-S curve during the domain breaking and vanishing is similar but opposite to that of domain nucleation in Fig. 2 during loading except that the former is on a much lower stress level. The typical domain size and load jump are also much less distinct (a smaller jump of $3 \mathrm{MPa}$ in nominal stress 
during domain annihilation compared with a large drop of $15 \mathrm{MPa}$ during nucleation). Again, the competition between the domain front energy and strain energy played an important role in the domain morphology evolution of this stage.

3.7 Path dependence of patterns during unloading and reloading within the loop

It is worth checking the morphology and response of the tube for the unloading and reloading processes within the loop. Strong loading path dependence of the tube morphology was found and they are only briefly summarized as follows.

If the tube was unloaded before the domain growth reached its steady state where the mean contour of the branched front became perpendicular to the loading axis, the domain morphology was found to be reversible, i.e. the domain would evolve back to a helix rather than to a ring (Fig. 14(a)).

If the tube was unloaded after the domain growth reached its steady state, it can be seen clearly in section 3.5 that during unloading the cylindrical domain would not reversibly evolve back to the helical domain, instead, it would evolve to the inclined smooth ring-like domain (also see Figs. 12 and 13). If the tube with such a ring-like domain was reloaded to the upper stress plateau, the domain would first grow in a stable manner and then its smooth front branched macroscopically at the length of $1.20 \mathrm{~mm}$ which was a little larger than that for unloading ( $\approx 1.10 \mathrm{~mm})$, see Fig. 14(b). 
If the tube with a C-shaped domain (see $h \sim m$ of Fig. 13) was reloaded to the upper stress plateau, the $\mathrm{C}$ shape would not heal up to its original ring. Instead, it would grow into a helical domain along one of its more favored tip direction at an orientation of about $60^{\circ}$ as shown in Fig. 14(c), while the other energetically less favored tip would serve as a kind of defect which basically does not influence the helical growth and would eventually be engulfed by the helical domain.

We would like to end this section by emphasizing the important influence of structure-material coupling in the above domain evolution. Such coupling, in the form of the created torsion and bending in the tube, is unavoidable during the tube elongation. For example, the asymmetric domain patterns either at the initial domain formation or at the subsequent domain growth will lead to an asymmetric torsion and bending in the tube, which in turn will influence the domain pattern and evolution. Though the whole process is still dominated by tension for the thin-walled long tube, transformation under axial stretching would simultaneously produce torque and a bending moment in the tube. Both are expected to vary with domain growth and be detected. In the present research special attention was paid to the torque response during stretching. However, the measurement of such a small torque has not been successful in the experiment because of the considerably low torsion rigidity of the 
thin-walled long microtubing. This data gap will be filled by continued effort in the future.

\section{Discussions of the Experimental Results}

From the results of section 3, we can see that there are basically two types of instability events which took place during the transformation process of the tube. One is the instability of a homogeneous deformation, which leads to the phenomenon of band nucleation and vice versa, band annihilation (see Fig. 4 and Fig. $13 h \sim n$ ). The other is the instability of the existing domain wall, which leads to interface branching and domain morphology transition (see Figs. (8), (9), (12) and (13) $a \sim g$ ). Both events are dynamic (i.e. in an uncontrollable manner) even under extremely small nominal strain rate and they both occupy distinctly much shorter time spans than that of the stable propagation of the front (notice the time coordinates in the figures mentioned above). If we examine further the stable propagation of the macroscopic front with even higher resolution, it would again contain numerous distinct and successive micro-instabilities associated with the nucleation, annihilation and switching of microscale domains inside individual grains in the narrow frontal zone. Such multi-scale spatiotemporal scenario of the deformation process reflects the intrinsic discrete natures of the material hierarchy of the polycrystal. Therefore the above 
processes are all dissipative and are governed by different kinetics of the material.

They will be discussed in the following.

4.1 Physical origin of the macroscopic deformation instability in polycrystals

For the first type of instability (instability of the homogeneous deformation), it is seen from Fig. 2 that before the macroscopic domain nucleation the loading curve exhibited small nonlinearity due to the formation of many microscopic martensite bands in the polycrystal but the macroscopic deformation is still homogeneous. Such small scale transformation is evidenced by both the small hysteresis loop upon unloading (see Fig. 3) before the peak load and the uniform temperature increase of about $0.2^{\circ} \mathrm{C}$ at the sample surface. This homogeneous deformation (here termed as pre-burst incubation) persisted until the peak load that marks the beginning of macroscopic burst transformation. The pre-burst (macroscopically stable) and burst-like (autocatalytic) transformations (as described in sub-stages (i) (iii) in detail in section 3.2) are typical of fine grained NiTi polycrystals in tension (see Li and Sun, 2002, Sun and Li, 2002 for microtubes; Shaw and Kyriakides, 1995, 1997 and Sun et al., 2001 for strips and wires). They were also observed in the transformation of other systems (see Nishyama, 1978; Reyes-Morel and Chen, 1988; Rose and Swain, 1988 for ceramics; and Yu and Clapp, 1989 for metals, among others). They were discussed 
in the literature using various terminologies such as autocatalytic effect, burst, avalanche, chain reaction, percolation, etc.

There are two explanations for the possible physical mechanisms of the macroscopic deformation instability shown in Fig. 2. One speculated mechanism is the autocatalytic nucleation and coalescence of many micro-martensite bands or variants inside very small grains of the NiTi polycrystal. The autocatalytic transformation happened because the interaction among the local stress fields of these micro-domains formed in the pre-burst stage become increasingly strong with loading and eventually these micro-events percolate up to the macroscopic level and lead to the strain softening of a macroscopic representative material element until it is fully transformed. The phenomenon of autocatalysis, wherein a region of elastic phase transformation creates stresses sufficient to drive alone further transformations, was identified long time ago in the material systems mentioned above. In real polycrystals there is always a competition between the softening mechanism and the hardening mechanism (e.g. due to grain orientation) in the overall behavior. It is strongly influenced by the detailed microstructure (e.g. texture and grain size distribution) and the stress state (e.g. tension, shear or compression). In terms of elasticity, the softening stress-strain relation corresponds to a macroscopic non-convex strain energy function of the material during phase transition. Another possible mechanism leading 
to the macroscopic band formation, without invoking the intrinsic material softening, is the geometric instability due to the finite transformation strain. This is similar to the neck formation observed in some polymers where the material's true stress-strain relation is still stable. The investigation on this type of localized deformation has been well documented in the literature (see review articles by Needleman and Tvergaard, 1992; Nguyen, 1994, Tomita, 1994; Petryk, 1997 and the large number of references therein). Either geometric instability (for NiTi, see Orgeas and Favier, 1998, Favier et al., 2001, Sittner et al., 2005) or material instability (Falk, 1980, 1982, 1983, Barsch and Krumhansl, 1988, Abeyaratne et al., 2001), it is usually not straight forward from the measured nominal S-S curve to see immediately which one is dominant for the domain formation in the NiTi polycrystal.

According to some micromechanical models (Roitburd, 1973; Sun et al., 1991; Sun and Hwang, 1993; Bruno et al., 1996; Smyshlyaev and Willis, 1998) the elastic energy of a transforming polycrystal can be non-convex (softening stress-strain relation) for certain types of microstructures. One of the simplest form of non-convex energy is the $A f(1-f)$ type, where $A$ is a constant, $f$ is the volume fraction of a cluster of small spherical (circular in 2D) martensite variant for fine grained polycrystal. The material used in this paper is indeed extra-fine grained polycrystal (grain size of 50 100 nm). Another argument supporting the strain softening in tension is the 
measured value of load drop during macroscopic domain formation. It has been shown (Shaw and Kyriakides, 1998) that geometric instability alone (due to the finite $5 \%$ transformation strain of the domain) can only cause a relatively small load drop. Using the values of modulus and strains from the tube, the maximum allowable load drop due to geometric instability (with stable tri-linear stress-strain relation) is only about 23.4 MPa, which is much less than the experimental observation of 42MPa 90MPa (Tse and Sun, 2000; Li, 2002). The non-convexity of strain energy function for the polycrystal is also supported by recent finite element simulation (He and Sun, 2005) on domain morphology in NiTi tube. The results indicated that only geometry effect is not sufficient to quantify the experimental observation and that material instability dominates the observed helical domain formation.

There have been notable theoretical efforts in recent years to model the nucleation peak, propagation plateau and hysteresis phenomena, emphasizing the discreteness and nonlocality of the phase transition process (see Abeyaratne et al., 1996; James, 1996; Ortin and Delaey, 2002; Triantafyllidis and Bardenhagen, 1993; Truskinovsky and Vainchtein, 2004 and the references therein). The present observations appear to be consistent to such a picture that microscopic instability developed at the macroscopic homogeneous deformation stage and continues after the macroscopic system becomes unstable. More detailed systematic measurement and 
quantitative modeling for real polycrystals along this line of thought remain to be conducted in the future.

4.2 Macroscopic domain wall energy and its role in morphology evolution

For the second type of instability (instability of the domain wall), it is basically attributed to the differences in the total energy (bulk strain energy and interfacial energy) among the possible states of domains in tube system. Such difference leads to morphology transition. It bares lots of similarities with the morphological instabilities in other solid systems (for example, see Srolovitz, 1989 and Suo, 1997). Here the existence of macroscopic domain wall energy (interfacial or front energy) and its role in the formation and evolution of domains are strongly indicated by the present research. Although the idea of energy associated with the macroscopic transformation front is not new, it is almost completely omitted in the previous research on instability of polycrystalline NiTi. For the purpose of simplicity, we purposely treat the domain structure as the minimizer of free energy of the actually dissipative. According to such equilibrium frame work the domain formation and evolution in the tube configuration can be understood as follows.

First, it is seen that the formation and growth of the helical domain broke the symmetry of axial loading and axial symmetric tube geometry. This symmetry-breaking morphology and its subsequent evolution could be attributed to the 
energy preference among “possible equilibrium shapes” of a domain inside which the transformation strain is shear in nature. The difference in the dependence on domain size of bulk energy (such as elastic strain energy) and the domain wall energy will make the equilibrium domain shape also size-dependent (Johnson and Cahn, 1984; Jog et al., 2000; Voorhees and Johnson, 2004). For example, during the nucleation of the macroscopic helical domain the energy of the domain wall played an important role for the finite aspect ratio of the domain, otherwise its shape would be unrealistically long and narrow. The domain self-merging during loading and annihilation during unloading could also be understood according to such interplay between the two energies. When the two fronts come close enough the driving force from the rapid reduction in the interfacial energy of the system accelerated the front merge, producing an obvious load change in the S-S curve. The role of front energy manifested again during the formation of the branched front and front smoothing.

Second, the competition between bulk and interfacial energies during unloading/reloading can lead to branched ring $\leftrightarrow$ smooth ring and smooth ring $\leftrightarrow$ inclusion topology transitions. It is noticed that the helix (a lenticular inclusion once flattened) and ring (inclined narrow cylinder) are the two basic but topologically different forms of the domain in the tube configuration. The ring-like domain is sandwiched by the two separated austenite (lower strain phase) while the helical band 
is a single inclusion embedded in the austenite. Compared with the cylindrical domain of the same volume, the helix has relatively larger interface area but has much less elastic strain energy. Therefore, to minimize the total energy, a helical domain is preferred to form when the interfacial energy density is relatively small, while a ring domain is preferred to form when the interfacial energy density is relatively large (see $\mathrm{Ng}$ and Sun, 2006). From thermodynamics point of view, the reported topology transition and its loading path dependence reflect the multiplicity of metastable domains in the tube configuration.

Third, regarding the physical nature of the macroscopic scale domain wall energy in polycrystal, it is very similar to the energy of the interface (habit plane) between austenite and twinned martensite in single crystal due to local incompatibility (Fig. 15(a)) (among many literatures, see Khachaturyan, 1983, Roitburd, 1998, and recently Maciejewski et al., 2005). One of the key complexities in quantifying this energy is the hierarchy of the macroscopic domain wall in polycrystal as shown in Fig 15 (b). It is seen that a macroscopically compatible "sharp" front, after zoom in, is in fact a very incompatible zone containing many differently oriented grains and micro-domains. The local stress field of this interfacial zone is highly non-uniform. It is also seen that for the nano-grained polycrystal of this paper, $l_{p}$ should be much smaller than the value measured by surface profiler in Fig. 6. Experimental study to 
quantify the value of $l_{p}$ is currently under way. For a pure phenomenological purpose, the front energy could be estimated by fitting the morphology data with the calculation using the nonlocal and nonconvex framework (Cahn and Hilliard, 1958; Falk, 1983).

We should finally emphasize that the interaction between material and structure geometry always exists and sometimes even strongly influences the observed phenomena of morphology evolution in the tube system through the created torque and bending moment. A quantitative account of this aspect will rely on future modelling and simulation.

4.3 Mobility of the front and heat effect during domain pattern evolution

In the present tube configuration a single helical domain could survive and grow after nucleation so that a nominal stress and front velocity relationship could be obtained. This measured relationship captured the macroscopic effect of those micro-thermomechanical events which facilitated the macroscopic front motion in polycrystals (Abeyaratne and Knowles, 1990, 1997). Usually a front kinetics expressed as $V=V(F)$, relating the front velocity $V$ and the thermodynamic driving force $F$ at the front, is supposed to exist and is proposed as an intrinsic constitutive function of the material. Under an externally applied stress the driving force of the front can be calculated. In tube configuration the faster growth of the initial domain at 
the tip than that in transverse direction turned the domain into a helical shape. In extracting the isothermal front kinetics $V=V(F)$ from the measured data the heat effect which results from generation, conduction and convection of latent heat must be carefully taken into account.

It is known that the latent heat generated as the front propagates during $A \rightarrow M$ transition will reduce the chemical driving force, so the front velocity is limited by heat transfer. At the same time, as the domain grows, the resulted load drop will also reduce the mechanical driving force of the front, accompanied by a rapid decrease in front velocity as shown in Figs. 4 and 16. The two stages in the load drop process described in section 3.2 and Fig. 4 served to demonstrate respectively the influences of the above two factors in the front motion. In a one-dimensional setting, such thermomechanical coupling in the measured nominal kinetic relationship has been analyzed by several researchers (see Bruno et al., 1995; Shield et al., 1997 and Shaw, 2002). In their work, by considering the steady state motion of a single interface in the wire and taking into account the latent heat generated during front propagation, a closed-form relationship between the steady-state interfacial speed and the overstress (i.e. the difference between the applied stress and the plateau stress) was derived (Bruno et al., 1995 and Shield et al., 1997). As shown in Fig. 16, using the material constants for NiTi, our measured results $\left(V_{m}\right)$ at the middle point of the long front 
agree quite well with the $1 \mathrm{D}$ theoretical relation (notice that the mechanical driving force at the middle point of the front is approximately equal to the external applied stress). The measured temperature rise due to the latent heat for the range of front velocity in the present test is within $2^{\circ} \mathrm{C}$ which is also close to the results of Leo et al., (1993) for wires under similar front velocities. However, for the same externally applied nominal stress, the tip velocity $V_{t}$ (Fig. 16) is much higher than $V_{m}$. Whether this is due to a higher mechanical driving force at the tip (than at the middle point of the front) or simply due to the geometric compatibility requirement of the cusp-shape domain boundary (see Fig. 7 where the driving force might be still the same as that in the middle point of the front) needs to be clarified in the future.

Research on the macroscopic front kinetics of polycrystalline NiTi (see Leo et al., 1993; Bruno et al., 1995 and Shield et al., 1997) and Cu-Al-Ni single crystals (Abeyaratne et al., 1996; Abeyaratne and Knowles, 1997; Escobar and Clifton, 1993; Grujicic et al., 1985) demonstrated that the kinetic function has some common characteristics with those of frictional behavior in that a small change in the driving force will lead to a rapid change in front velocity. Because of the strong similarity between dislocation and displacive transformation ("martensitic") at the microscopic scale, there are strong parallels between the kinetics of displacive transformation and plastic deformation (Ashby and Jones, 2001). This means that for isothermal 
quasistatic front motion, the total driving force (i.e. Maxwell stress plus the dissipative force (in loading)) is independent of the front speed, or the speed of the front is exactly what is required for the front to have a constant driving force. Thus for the quasistatic motion of a macroscopic front here (front velocity of the order of 10 $\mathrm{mm} / \mathrm{s}$ ) the intrinsic macroscopic front kinetics $F=F(V)$ could be treated as the rate-independent type or perfect-plasticity type. The measured apparent stress-dependency of the front velocity is mainly a manifestation of the thermal effect which is masked onto the intrinsic isothermal kinetics. Such a picture is supported by the current experiment even though the influence of other factors (such as material anisotropy) on the domain morphology and front mobility has not been taken into account. As the summary of this section, the roles of energetics and kinetics as well as their interplay in governing the observed domain evolution are schematically drawn in the Fig. 17.

\section{Conclusions}

Taking advantage of the tube configuration and using the synchronized load-deformation and surface morphology observation technique, we were able to observe and record the whole transformation process, from nucleation to growth and to the eventual vanishing, of a single stress-induced macroscopic deformation domain in the tube specimen under displacement controlled uniaxial tension. The observation 
revealed several interesting pictures of spatiotemporal evolution of this domain during loading, unloading and reloading. Important processes and events such as pre-burst incubation, dynamic domain formation, front branching, front convolution, front instability and domain topology transitions were recorded and measured quantitatively. This research brought up several critical issues for phase transition in polycrystalline materials under mechanical forces. The underlying physical mechanisms and the related modeling aspects have been discussed. Some of them can be understood readily but others are still quite vague or speculative and need further investigation. The conclusions of the paper are listed as follows:

- The domain morphology evolution during the loading process can be summarized as the sequence of from the dynamic formation of the helical domain to its further growth and its front branching, then to the self-merging and topology transition into a cylindrical domain, and finally to the stable growth of the cylinder-like domain by the convoluted motion of its branched front.

- The domain pattern evolution during unloading is not a simple reversal of that in the loading process but exhibits rather strong loading history dependence. For monotonic unloading the pattern evolution is basically via the sequence of from the reverse convolution of the branched front to the switching into smooth front, and then to domain breaking and eventually to dynamic domain annihilation. 
- Even under the extremely small nominal strain rate, the phase transition process still involves both macroscopic static (such as stable growth of domain with very slow front motion) and macroscopic dynamic events (such as front branching / smoothing and domain formation/merging/annihilation). The whole deformation period is divided into two distinct macroscopic time spans associated with the stable propagation and unstable macroscopic front propagation respectively. The latter is much shorter than the former. The former, if looked with even higher resolution, would be again realized through the numerous successive microscale nucleations, growth and switching inside the macroscopic frontal zone. The macroscopic part of this multi-scale process in the polycrystalline material is observed in the present experiment.

- The analysis of the observed phenomena in tube configuration clearly reveals that the competition between the macroscopic domain wall energy and the bulk energy played an important role in domain morphology evolution. The origin of the domain wall energy is attributed to the intrinsic heterostructures of the polycrystal. The domain wall thickness and the effective wall energy are expected to be proportional to the characteristic length of the heterostructures. Quantifying this energy term in polycrystalline materials remain open for future investigation.

- The velocity of the front during dynamic domain formation is measured directly in 
the experiment. The obtained nominal overstress and front speed relationship supports the isothermal rate-independent or perfect-plasticity type kinetics for the quasistatic front motion. Such relationship also agrees with the previous theoretical model prediction which is based on a one-dimensional setting taking the heat transfer effect into consideration. It is the combined effort of kinetic law and energetic preference that governs the various observed aspects of domain formation and evolution.

Acknowledgement: The authors are grateful for the financial support from the Research Grants Council of the Hong Kong SAR, China (through Projects HKUST 6234/01E, HKUST 6245/02E and HKUST 6156/04E). QPS is grateful to the French Ministry of Education for financing a visit at LMT (CNRS) of Ecole Normale Superieure de Cachan in Paris hosted by Prof. Han ZHAO. He also would like to thank Prof. K. Bhattacharya of the California Institute of Technology, Prof. S. Kyriakides of the University of Texas at Austin and Prof. H. Petryk of IPPT of the Polish Academy of Science for the stimulating discussions on the results of this paper. 
Appendix A — Four subsystems of the developed testing system

The testing of microtubing requires a series of careful and precise operations, such as measurement under a large twist up to $720^{\circ}$, microscopy and profilometry to quantify surface morphology, high speed data acquisition for the dynamic domain formation, and synchronized recording of load response and surface images, etc. These operations are not supported by the existing universal testing machine system, so it is necessary to develop a testing system suitable for NiTi microtubing. As shown in Fig. A1, the testing system is mainly made up of four subsystems: the loading subsystem, measurement subsystem, surface observation subsystem and automation subsystem.

The loading subsystem is designed to apply a controlled loading to the tubing specimen. A stepping motor (HS2221, MAE, Italy) is installed to drive the system. Controlled by a microstepping drive (IM804, IMS, USA), the motor can run with a rotary angle resolution as small as $0.007^{\circ}$. The speed can be as slow as $0.001 \mathrm{rpm}$, corresponding to an elongation rate of $5 \mu \mathrm{m} / \mathrm{min}$ of the specimen when a ball screw with $5 \mathrm{~mm}$ lead (RNFTL1405A2.5S and RS1405A5, NSK, Japan) is used to transform the rotation into linear motion. This is ideal for quasi-static loading. The drive is connected to a computer with a motion control card (DMC1000, Leisai, China) which makes automatic and programmable loading possible. Thus, the motor can provide a smooth load in a controlled and quasi-static way. Tube grips are used to fix the two ends of the specimen. The friction between the grip and the specimen is large enough to guarantee that there is no sliding between them.

The measurement subsystem is to measure the nominal strains and stresses of the specimen. A combined sensor was designed by the authors and manufactured by a company (TML, Japan). In the alternative solution, a load cell (445 N, 2308-01A, PCB, USA) and a torque sensor (0.57 N.m, 1538-01A, PCB, USA) are employed. There are two strain measurement configurations (see Feng, 2005). In the single-sided configuration, one grip is fixed; the axial motion of the other grip is measured by a cantilever sensor (CE-5, TML, Japan) whose tip is in contact with the bottom of the grip; the rotary motion is measured by a dial sensor (DDP-30A, TML, Japan) whose tip is in contact with the wing plate fixed on the side of the grip. In the double-sided configuration, one grip is constrained against axial motion but free to rotate and the other is constrained against rotation but free to conduct axial motion; the installation of the sensors is similar.

The surface observation subsystem is to observe the surface of specimens. An imaging camera (TK-C1381EG, JVC, Japan with telescopic lens Zoom 7000, Navitar, USA) is employed to directly observe the surface morphology (such as high-strain 
domain shape and orientation, etc.) of the tube during loading and unloading. A microscope (zoom stereo, SZH-10, Olympus, Japan with digital camera Coolpix 4500, Nikon, Japan) is employed to obtain more detail of the surface morphology. A high speed motion camera (8000 fps max., Motion Scope PCI, Redlake, USA) is used to capture the moment of domain formation. It should be emphasized that lighting is essential to obtain high quality images. A profiler (vertical resolution $<0.3 \mathrm{~nm}$, NT3300, Wyko, USA) is used to quantify the in-plane and out-of-plane surface displacement and therefore the corresponding strains. An infra-red camera (PM695, FLIR, USA) is employed to evaluate the temperature distribution on the specimen due to the latent heat of the phase transformation. The size of the system must be restrained to allow observation by these apparatus.

The automation subsystem is to make automatic testing possible. Automatic loading is facilitated by a motion control card in a computer. Automatic measurement of the nominal strains and stresses are achieved mainly by a data acquisition card (UA302a, USBAD, China). All the sensors are connected to several signal conditioners (DC-92D, TML, Japan and F8159, PCB, USA). The conditioners are then connected to the data acquisition card which is linked to the computer. The data acquisition rate is set to about 100 readings per second for general purpose. The rate can be set to as high as 100,000 readings per second for dynamic processes such as domain formation. Automatic image acquisition from the imaging device of the observation subsystem is realized by a multi-function display card (Winfast A310 TD VIVO, Leadtek, Taiwan) in the computer. Software is written by the authors to define the loading process, to display the stress-strain curve and the morphology in real time, to synchronize data and image acquisition, to define data acquisition process, etc.. A complete system is shown in Fig. A-1.

\section{References}

Abeyaratne, R. and Knowles, J.K. (1990) On the driving traction acting on a surface of discontinuity in a continuum. J. Mech. Phys. Solids 40, 345-360.

Abeyaratne, R. and Knowles, J.K. (1993) A continuum model of a thermoelastic solid capable of undergoing phase transitions. J. Mech. Phys. Solids 41, 541-571.

Abeyaratne, R. and Knowles, J.K. (1997) On the kinetics of an austenite $\rightarrow$ martensite phase transformation induced by impact in a Cu-Al-Ni shape-memory alloy. Acta Mater. 45, 1671-1683.

Abeyaratne, R., Chu, C. and James, R. D. (1996) Kinetics of materials with wiggly energies: theory and application to the evolution of twinning microstructures in a CuAlNi shape memory alloy. Phil. Mag. A 73, 457-497. 
Abeyaratne, R., K. Bhattacharya, K. and Knowles J.K. (2002) in Nonlinear elasticity: theory and applications (ed. Y. Fu and R.W. Ogeden), Cambridge University Press, 433-490.

Ashby, M. F. and Jones, D. R. H. (2001) Engineering Materials 2, $2^{\text {nd }}$ edition, Butterworth Heinemann.

Barsch, G.R. and J.A. Krumhansl J.A. (1988) Nonlinear and nonlocal continuum model of transformation precursors in martensites. Metallurg. Trans. A 19, 761-775.

Bhattacharye, K. (2003) Microstructure of Martensite, Oxford Univ. Press.

Bhattacharye, K., Conti, S., Zanzotto, G. and Zimmer, J. (2004) Crystal symmetry and the reversibility of martensitic transformations. Nature 428, 55-59.

Berg, B. (1997) Twist and Stretch: Combined Loading of Pseudoelastic NiTi Tubing. Proc. Shape Memory and Superelastic Technologies 1997 (ed. A.R. Pelton, D.E. Hodgson, S.M. Russell, and T.W. Duerig), 443-448.

Brinson, L.C., Schmidt, I. and Lammering, R. (2004) Stress-induced transformation behavior of a polycrystalline NiTi shape memory alloy: micro and macromechanical investigations via in situ optical microscopy. J. Mech. Phys. Solids 52, 1549-1571.

Bruno O.P., Leo P.H., and Reitich F. (1995) Free boundary conditions at austenite-martensite interfaces. Phys. Rev. Lett. 74, 746-749.

Cahn, J. W. and Hilliard, J. E. (1958), Free energy of a nonuniform system I. Interfacial energy. The J. of Chemical Physics 28 (2), 258-267.

Escobar, J.C. and Clifton, R.J. (1993) On pressure-shear plate impact for studying the kinetics of stress-induced phase transformations. Mater. Sci. Eng. A 170, 125-142.

Falk F. (1980) Model free-energy, mechanics and thermodynamics of shape memory alloys. Acta Metallurgica 28, 1773-1780.

Falk F. (1982) Landau theory of martensitic phase transitions. Journal De Physique IV, C4 43, 3-15.

Falk F. (1983) Ginzburg-Landau theory of static domain-walls in shape-memory alloys. Z. Physik B Condensed Matter 51, 177-185.

Favier, D., Liu Y., Orgeas L. and Rio R. (2001) Solid Mechanics and Its Applications, Vol. 101, ed. By Q. P. Sun, Kluwer Academic Publisher, 205-212.

Feng, P. (2005) PhD thesis, Hong Kong University of Science and Technology, Hong Kong.

Feng, P. and Sun, Q. P. (2006) Identification and measurement of the domain strain in NiTi microtubing under biaxial loading, to appear in Smart Materials and Structures, 2006.

Gruijicic, M., Olson, B.B. and Owen, W.S. (1985) Mobility of the $\beta_{1}-\gamma_{1}^{\prime}$ martensite interface in Cu-Al-Ni: Part I. experimental measurements. Metal. Trans. A 16, 1723-1734.

He, Y.J. and Sun, Q.P. (2005) Proc. of Int. Conf. on Solid-Solid Phase Transformations in InoganicInorganic Mater., May 29-June 3, 2005, Phoenix, Arizona, USA.

James R.D. (1996) Wiggly Energies, the Symposium in honor of J. L. Ericksen, June 12-14, Maryland, USA.

Jog, C.S., Sankarasubramanian, R. and Abinandanan, T.A. (2000) Symmetry-breaking transitions in equilibrium shapes of coherent precipitates. J. Mech. Phys. Solids 48, 2362-2389. 
Johnson, W.C. and Cahn, J.W. (1984) Elastically induced shape bifurcations of inclusions. Acta metall. 32, 1925-1933.

Khachaturyan, A.G. (1983) Theory of structure transformation in solids, Wiley, New York.

Leo, P.H., Shield, T.W. and Bruno, O.P. (1993) Transient heat transfer effects on the pseudoelastic behavior of shape-memory wires. Acta metal. Mater. 41, 2477-2485.

Leo, P.H., Lowengrub, J.S. and Jou, H.J. (1998) A Diffuse Interface Model for Microstructural Evolution in Elastically Stressed Solids. Acta Mater. 46, 2113-2130.

Li, Z.Q. (2002) PhD thesis, the Hong Kong University of Science and Techology.

Li, Z.Q. and Sun, Q.P. (2000) Superelastic NiTi memory alloy micro-tube under tension - nucleation and propagation of martensite band. Key Engineering Materials 177-180, 461-466.

Li, Z.Q. and Sun, Q.P. (2002) The initiation and growth of macroscopic martensite band in nano-grained NiTi microtubes under Tension. Int. J. Plasticity 18, 1481-1498.

Maciejewski, G., Stupkiewicz, S. and Petryk, H. (2005) Elastic micro-strain energy at the austenite-twinned martensite interface. to appear in Archives of Mechanics.

Miyazaki, S., Imai, T., Otsuka, K. and Suzuki, Y. (1981) Lüders-like deformation observed in the transformation pseudoelasticity of a NiTi alloy. Scripta Metallurgica 15, 853-856.

Needleman, A. and Tvergaard, V. (1992) Analysis of plastic flow localization in metals. Appl. Mech. Rev. 45, S3-S18.

Nguyen, Q.S. (1994) Bifurcation and stability in dissipative media (plasticity, friction, fracture). Appl. Mech. Rev. 47, 1-31.

Ng, K.L. and Sun, Q.P. (2006) Stress-induced phase transformation and detwinning in NiTi tubes. Mechanics of Materials 38, 41-56.

Nishiyama, Z. (1978) Martensitic Transformation, Academic Press, New York.

Orgeas, L. and Favier, D. (1998) Stress-induced martensitic transformation of a NiTi alloy in isothermal shear, tension and compression. Acta Mater. 46, 5579-5591.

Ortin, J. and Delaey, L. (2002) Hysteresis in shape memory alloys. Int. J. Nonlinear Mechanics 37, 1275-1281.

Petryk, H. (1997) Plastic instability: Criteria and computational approaches. Arch. Comp. Mech. Eng. 4, 111-151.

Planes, A. and Manosa, L. (2001) Vibrational properties of shape-memory alloys. Solid State Physics 55, 159-267.

Reyes-Morel, P. E. and Chen, I. W. (1988) Transformation plasticity of ceria-stabilized tetragonal zirconia polycrystals: I, stress assistance and autocatalysis. J. Am. Ceram. Soc. 72, 343-353.

Rose, L. R. F. and Swain, M. V. (1988) Transformation zone shape in ceria-partially-stabilized zirconia. Acta Metall. 36, 955-962.

Shaw, J.A. and Kyriakides, S. (1995) Thermomechanical aspects of NiTi. J. Mech. Phys. Solids 43, 1243-1281.

Shaw, J.A. and Kyriakides, S. (1997) On the nucleation and propagation of phase transformation fronts 
in a NiTi alloy. Acta Mater. 45, 683-700.

Shaw, J.A. and Kyriakides, S. (1998) Initiation and propagation of localized deformation in elasto-plastic strips under uniaxial tension. Int. J. Plasticity 13, 837-871.

Shaw, J.A. (2002) A thermomechanical model for a 1-D shape memory alloy wire with propagating instabilities. Int. J. Solids and Struct. 39, 1275-1305.

Shield, T.W., Leo, P.H. and Grebner, W.C.C. (1997) Quasi-static extension of shape memory wires under constant load. Acta Mater. 45, 67-74.

Sittner, P., Liu, Y. and Novak, V. (2005) On the origin of Lüders-like deformation of NiTi shape memory alloys. J. Mech. Phys. Solids 53, 1719-1746.

Srolovitz, D. J. (1989) On the stability of surfaces of stressed solids. Acta Metall. 37, 621-625.

Sun, Q.P., Hwang, K.C. and Yu, S.W. (1991) A micromechanics constitutive model of transformation plasticity with shear and dilatation effect. J. Mech. Phys. Solids 39, 507-524.

Sun, Q.P. and Hwang K.C. (1993) Micromechanics modeling for the constitutive behavior of polycrystalline shape memory alloys I. derivation of general relations. J. Mech. Phys. Solids 41, $1-17,1993$.

Sun, Q. P. and Hwang K. C. (1994) Micromechanics constitutive description of thermoelastic martensitic transformations. Advances in Applied Mechanics (J. W. Hutchinson and T. Y. Wu ed.) 31, 249-298, Academic Press, New York.

Sun, Q.P. and Li, Z.Q. (2002) Phase transformation in superelastic NiTi polycrystalline micro-tubes under tension and torsion - from localization to homogeneous deformation. Int. J. Solids Struct. 39, 3797-3809.

Sun, Q.P., Li, Z.Q. and Tse, K.K. (2001) On superelastic deformation of NiTi shape memory alloy microtubes and wires — band nucleation and propagation. Proceedings of IUTAM symposium on smart structures and structronic systems, U. Gabbert and H. S. Tzou (eds.), Kluwer Academic Publishers, 113-120.

Suo, Z. (1997) Motion of microscopic surfaces in materials, Advances in Applied Mechanics, 33, 193-294, Academic Press.

Tomita, Y. (1994) Simulation of plastic instabilities in solid mechanics. Appl. Mech. Rev. 47, 171-205.

Truskinovsky, L. and Vainchtein, A. (2004) The origin of nucleation peak in transformational plasticity. J. Mech. Phys. Solids 52, 1421-1446.

Triantafyllidis, N. and Bardenhagen, S. (1993) On higher order gradient continuum theories in 1-D nonlinear elasticity: Derivation from and comparison to the corresponding discrete models. $J$. Elasticity 33, 259-293.

Tse, K.K. and Sun, Q.P. (2000) Some Deformation features of polycrystalline superelastic NiTi Shape memory alloy thin strips and wires under tension. Key Engineering Materials 177-180, 455-460.

Voorhees, P. W. and Johnson, W. C. (2004) The thermodynamics of elastically stressed crystals. Solid State Physics 59, 1-201.

Yu, Z.Z., and Clapp, P.C. (1989) Growth dynamics study of the martensitic transformation in Fe-30 Pct 
Ni alloys: Part I. Quantitative measurement of growth velocity. Metal. Trans. A 20, 1601-1615.

\section{Figure Captions}

Figure 1 Geometry of the dog-bone shaped sample: (a) schematic of the sample ( $L_{0}$ - length of the specimen, $L$ - gauge length, $L_{i}$ —end length in tube grip); (b) magnified regions of (a) ( $D_{0}$ - outer diameter of the as-received tube, $d$-inner diameter of the as-received tube, $D$ - outer diameter of the gauge section)

Figure 2 The whole process of tube deformation under quasi-static tensile loading and unloading: (a) the nominal stress-strain curve; (b) the corresponding surface morphologies

Figure 3 Stress loop indicating the occurrence of homogeneous phase transition before formation of a macroscopic domain: (a) unloading at strain 1.16\%; (b) unloading at strain $1.23 \%$

Figure 4 Formation and initial growth of the macroscopic domain (stage Loading-II): (a) the nominal stress-strain curve; (b) the corresponding surface morphologies

Figure 5 Domain formation observed by high speed camera: (a) the nominal stress-strain curve; (b) the corresponding surface morphologies

Figure 6 Surface profile of the helical domain corresponding to $C$ in Fig. 2

Figure 7 Front tip and mid-point velocity during domain formation: (a) the $V_{t}-t$ or $V_{t}-\varepsilon$ curve; (b) the $V_{m}-t$ or $V_{m}-\varepsilon$ curve; (c) the $V_{t}-\Delta \sigma$ curve; (d) the $V_{m}-\Delta \sigma$ curve

Figure 8 Self-merging of the helical domain: (a) the nominal stress-strain curve; (b) the corresponding surface morphologies; (c) 3D schematics of the morphology transition corresponding to $E$ in Fig. 2 and $d, e, f$ in (b) (light gray = high strain domain, dark gray = low strain domain); (d) unfolded plane view to illustrate the topology transition

Figure 9 Geometry of the branched front: (a) front view and back view of the tube surface; (b) the contour of (a) (light gray = high strain region, dark gray = low strain region); (c) 3D view of the front; (d) unfolded plane view of the front corresponding to (b) $\left(\theta_{1}=72^{\circ}, \theta_{2}=63^{\circ}\right)$

Figure 10 Surface profile of a region before and after phase transformation: (a) before deformation-stress-free austenite region; (b) after transformation-high strain martensite region inside the domain; (c) 
the positions of the same particles on tube surface corresponding to (a) and (b); (d) calculation of the axial strain $\varepsilon_{x}$ and the invariant-line angle $\theta_{0}$

Figure 11 Convoluted motion of the domain fronts: (a) a series of photos of tube surface; (b) the contour of (a) with cross marks and arrows showing the motion of one front tip; (c) comparison of energetics between a domain with smooth perpendicular fronts and a domain of the same volume but with branched fronts (light gray $=$ high strain region, dark gray $=$ low strain region)

Figure 12 Unsteady morphology evolution of the cylindrical domain with branched fronts gradually to an inclined smooth ring-shaped domain: (a) a series of photos of tube surface; (b) 3D schematics of the motion of the front on right-hand side (light gray = high strain region, dark gray = low strain region); (c) photos taken during front smoothing

Figure 13 Ring-like domain shrinkage and domain annihilation (stage Unloading-II): (a) the nominal stress-strain curve; (b) the corresponding surface morphologies; (c) 3D schematics (light gray = high strain region, dark gray = low strain region)

Figure 14 The stress-strain curves and corresponding surface morphologies during unloading and reloading within the loop: (a) unloading the tube with a helical domain; (b) reloading the tube with a ring-like domain; (c) reloading the tube with a C-shaped domain

Figure 15 Multi-scale structure of the austenite-martensite interface (front zone) and the material hierarchy therein (a) interface and interface energy in single crystal; (b) domain and domain energy in polycrystal tube (light gray $=$ high strain region, dark gray = low strain region)

Figure $16 \quad$ Variation of front velocity with stress drop

Figure 17 The interaction among domain morphology, driving force, and front motion (light gray = high strain region, dark gray = low strain region)

Figure A-1 Experimental setup (with the blueprint of the loading frame) 


\begin{tabular}{|c|c|c|c|c|c|}
\hline \multicolumn{3}{|r|}{ Loading } & \multicolumn{3}{|c|}{ Unloading } \\
\hline Stage & $\begin{array}{l}\text { Nominal } \\
\text { strain (\%) }\end{array}$ & Morphology & Stage & $\begin{array}{l}\text { Nominal } \\
\text { strain (\%) }\end{array}$ & Morphology \\
\hline $\mathrm{I}$ & $0 \sim 1.28$ & $\begin{array}{c}\text { Elastic deformation of } \\
\text { austenite and homogeneous } \\
\text { phase transition }\end{array}$ & I & $0 \sim 0.64$ & $\begin{array}{l}\text { Elastic deformation of } \\
\text { austenite }\end{array}$ \\
\hline II & $1.28 \sim 1.32$ & Domain formation & II & $0.64 \sim 0.69$ & $\begin{array}{c}\text { From ring-like domain to } \\
\text { C-shaped domain and } \\
\text { domain annihilation }\end{array}$ \\
\hline III & $1.32 \sim 3.19$ & $\begin{array}{c}\text { Helical domain growth and } \\
\text { self-merging }\end{array}$ & III & $0.69 \sim 0.86$ & $\begin{array}{l}\text { From cylindrical domain } \\
\text { with branched fronts to } \\
\text { smooth ring-like domain } \\
\text { and its shrinkage }\end{array}$ \\
\hline IV & $3.19 \sim 6.42$ & $\begin{array}{l}\text { Cylindrical domain growth } \\
\text { via front convolution }\end{array}$ & IV & $0.86 \sim 5.85$ & $\begin{array}{c}\text { Cylindrical domain } \\
\text { shrinkage via reverse front } \\
\text { convolution }\end{array}$ \\
\hline $\mathrm{V}$ & $6.42 \sim 6.77$ & $\begin{array}{c}\text { Elastic deformation plus } \\
\text { possible detwinning of } \\
\text { martensite }\end{array}$ & $\mathrm{V}$ & $5.85 \sim 6.77$ & $\begin{array}{c}\text { Elastic deformation of } \\
\text { martensite }\end{array}$ \\
\hline
\end{tabular}

Table 1 Stages of deformation during the loading and unloading processes 


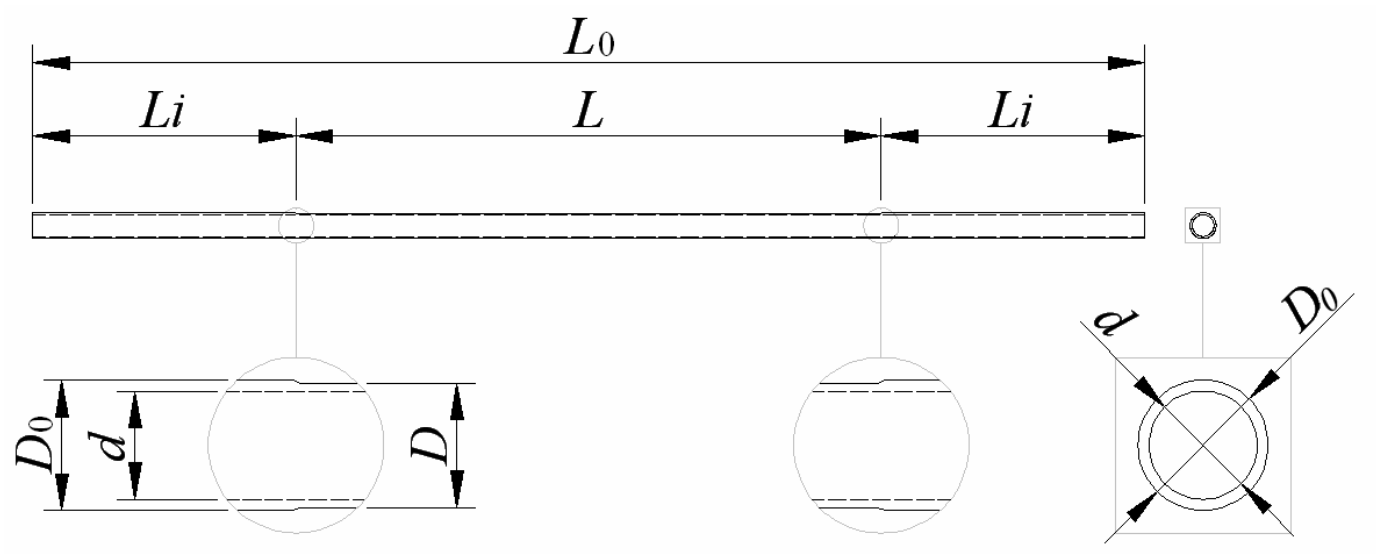

(a)

Figure 1 Geometry of the dog-bone shaped sample: (a) schematic of the sample ( $L_{0}$-length of the specimen, $L$ —gauge length, $L_{i}$ - end length in tube grip); (b) magnified regions of (a) ( $D_{0}$ - outer diameter of the as-received tube, $d$-inner diameter of the as-received tube, $D$ —outer diameter of the gauge section) 


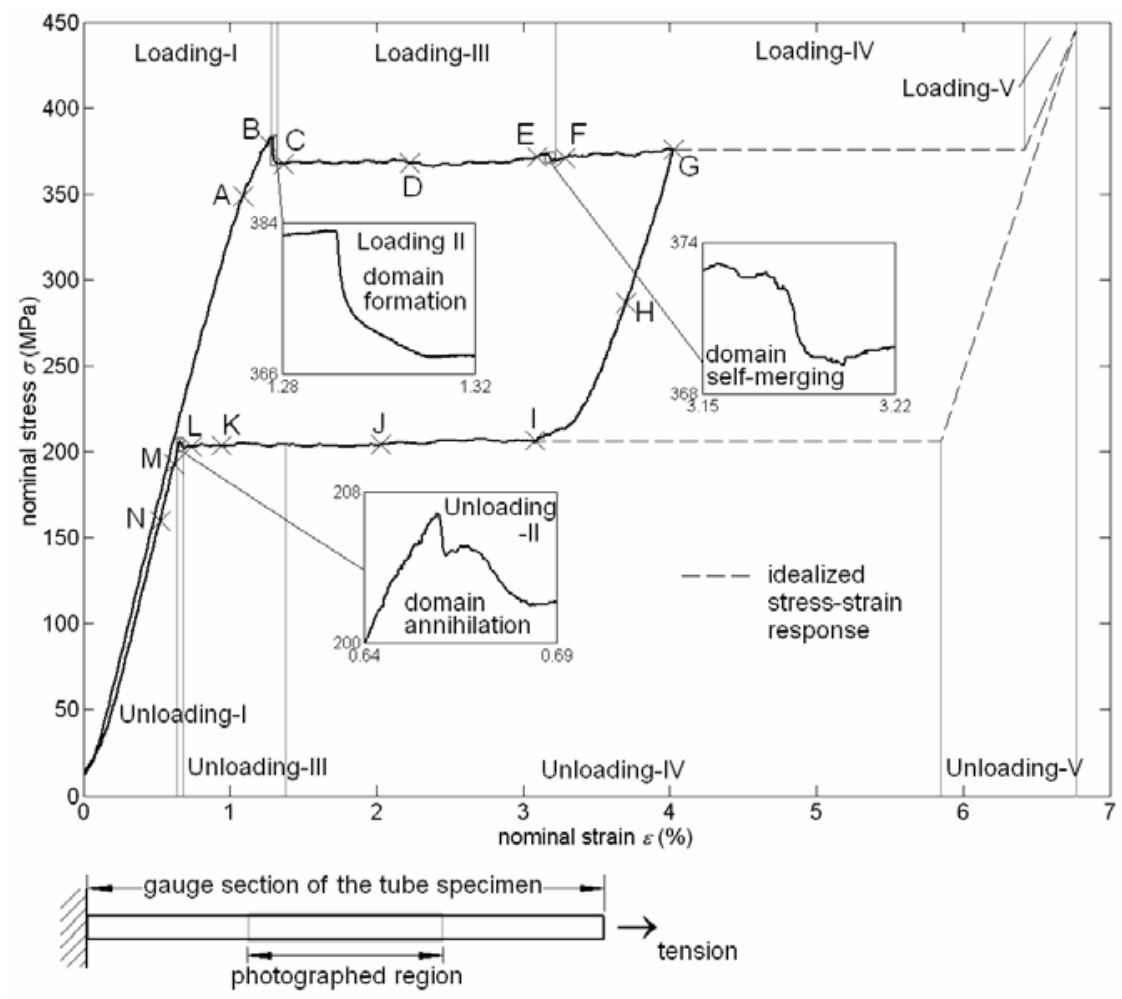

(a)

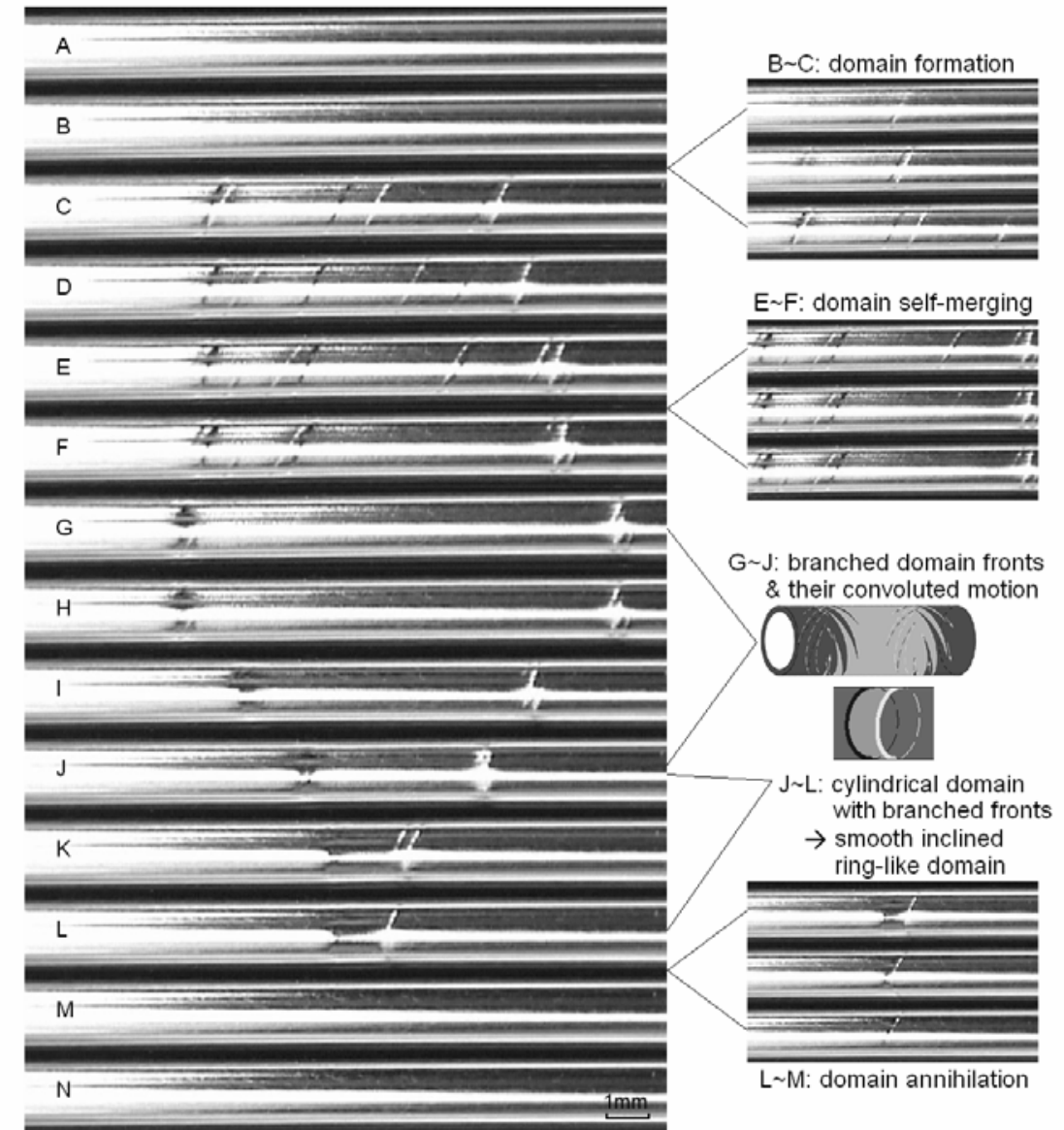

(b)

Figure 2 The whole process of tube deformation under quasi-static tensile loading and unloading: (a) the nominal stress-strain curve; (b) the corresponding surface morphologies 

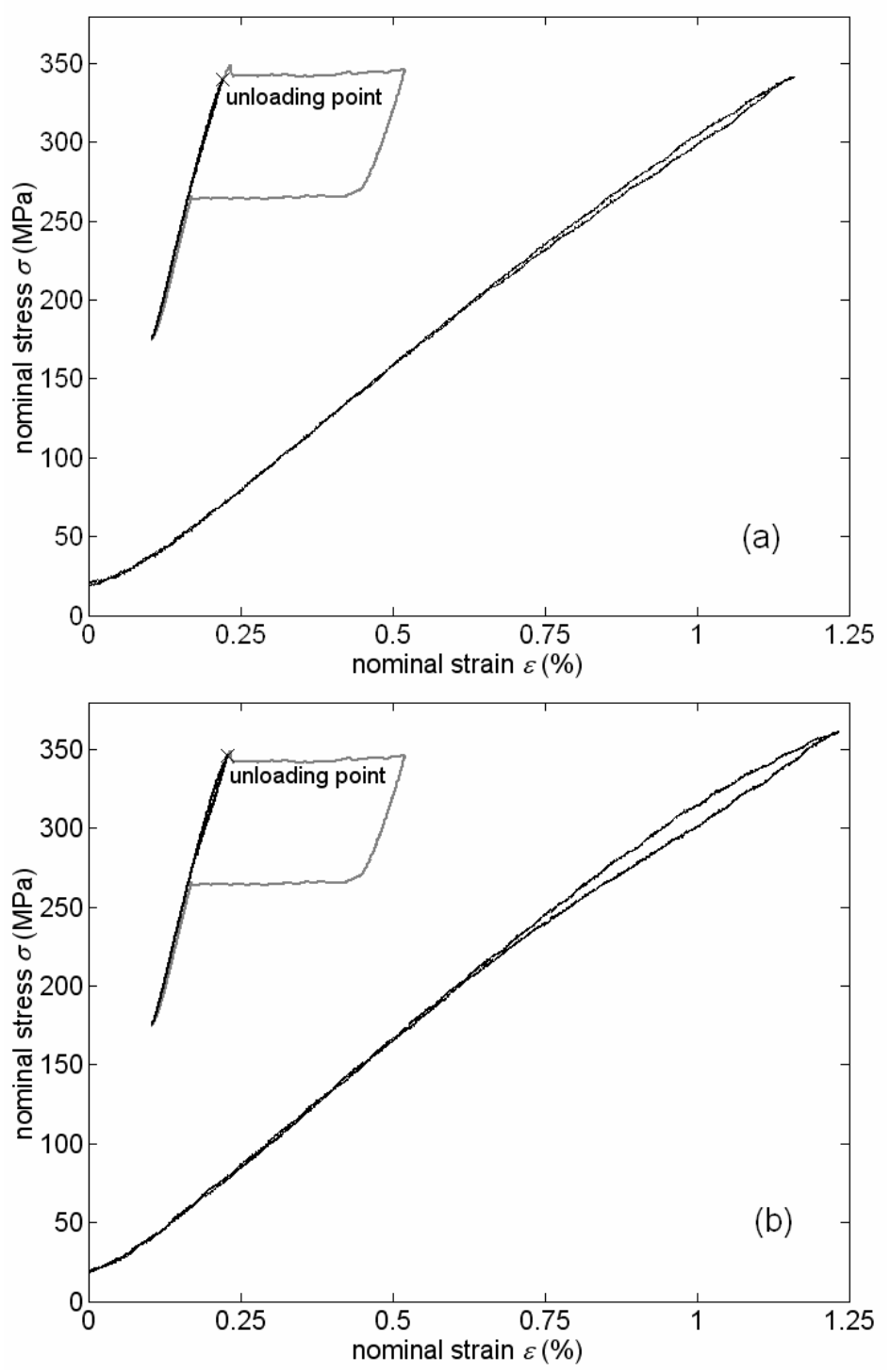

Figure 3 Stress loop indicating the occurrence of homogeneous phase transition before formation of a macroscopic domain: (a) unloading at strain 1.16\%; (b) unloading at strain $1.23 \%$ 


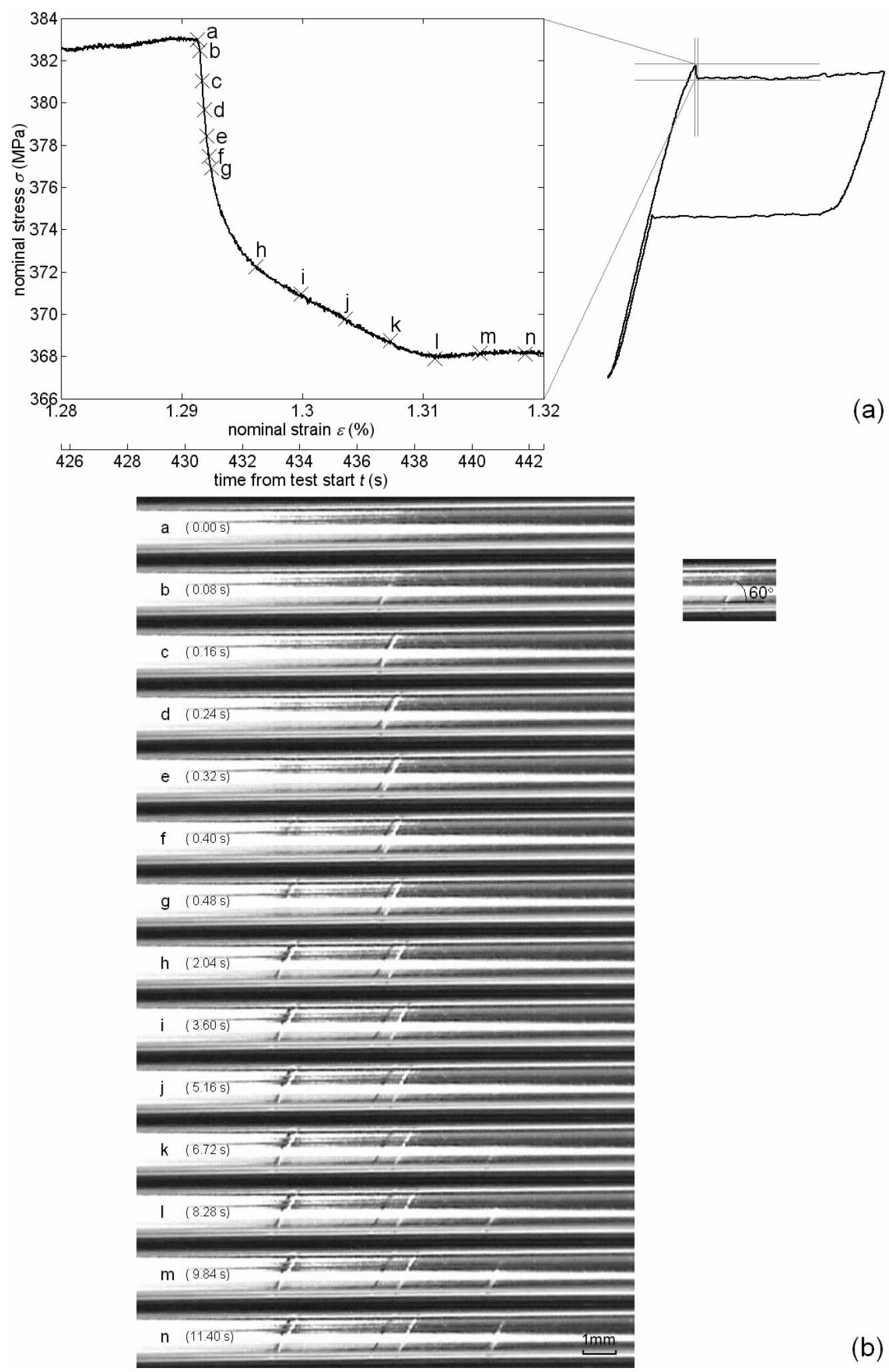

Figure 4 Formation and initial growth of the macroscopic domain (stage Loading-II): (a) the nominal stress-strain curve; (b) the corresponding surface morphologies 


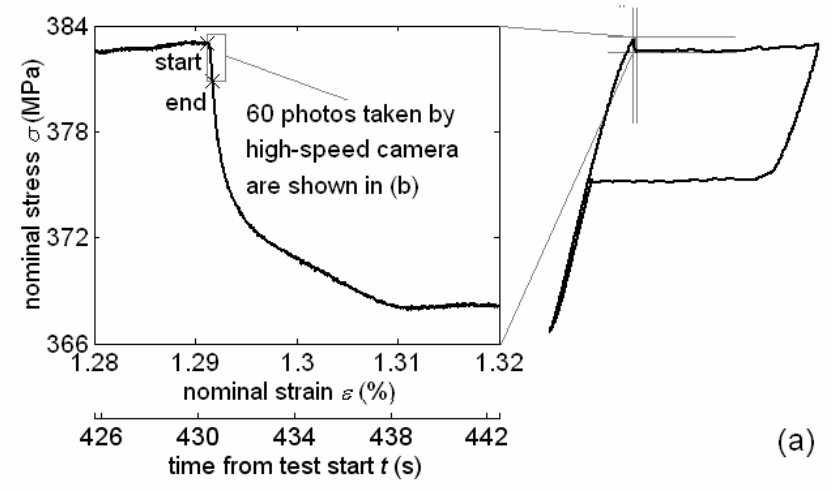

\begin{tabular}{|l|l}
\hline $0.000 \mathrm{~s}$ \\
\hline $0.004 \mathrm{~s}$ \\
\hline $0.008 \mathrm{~s}$ \\
\hline $0.012 \mathrm{~s}$ \\
\hline $0.016 \mathrm{~s}$ \\
$0.020 \mathrm{~s}$ \\
\hline $0.024 \mathrm{~s}$ \\
\hline $0.028 \mathrm{~s}$ \\
\hline $0.032 \mathrm{~s}$ \\
\hline $0.036 \mathrm{~s}$ \\
\hline $0.040 \mathrm{~s}$ \\
\hline $0.044 \mathrm{~s}$ \\
\hline $0.048 \mathrm{~s}$ \\
\hline $0.052 \mathrm{~s}$ \\
\hline $0.056 \mathrm{~s}$ \\
\hline $0.060 \mathrm{~s}$ \\
\hline $0.064 \mathrm{~s}$ \\
\hline $0.068 \mathrm{~s}$ \\
\hline $0.072 \mathrm{~s}$ \\
\hline $0.076 \mathrm{~s}$ \\
\hline
\end{tabular}
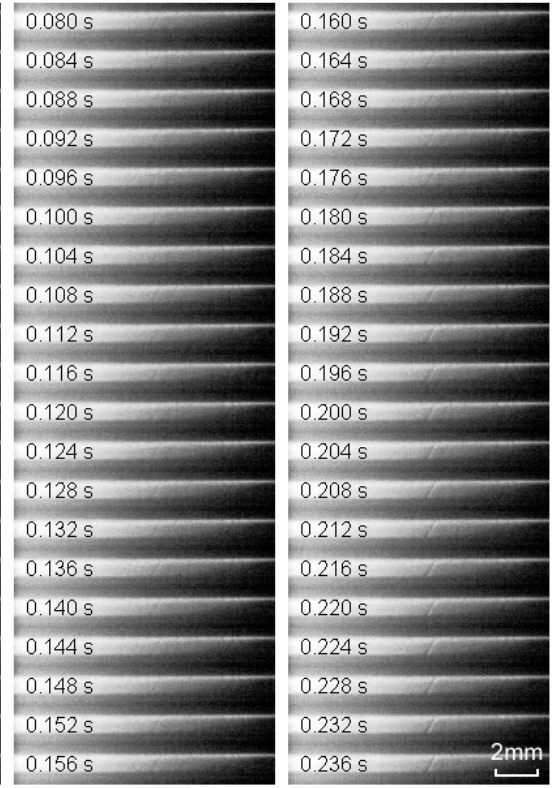

(b)

Figure 5 Domain formation observed by high speed camera: (a) the nominal stress-strain curve; (b) the corresponding surface morphologies 


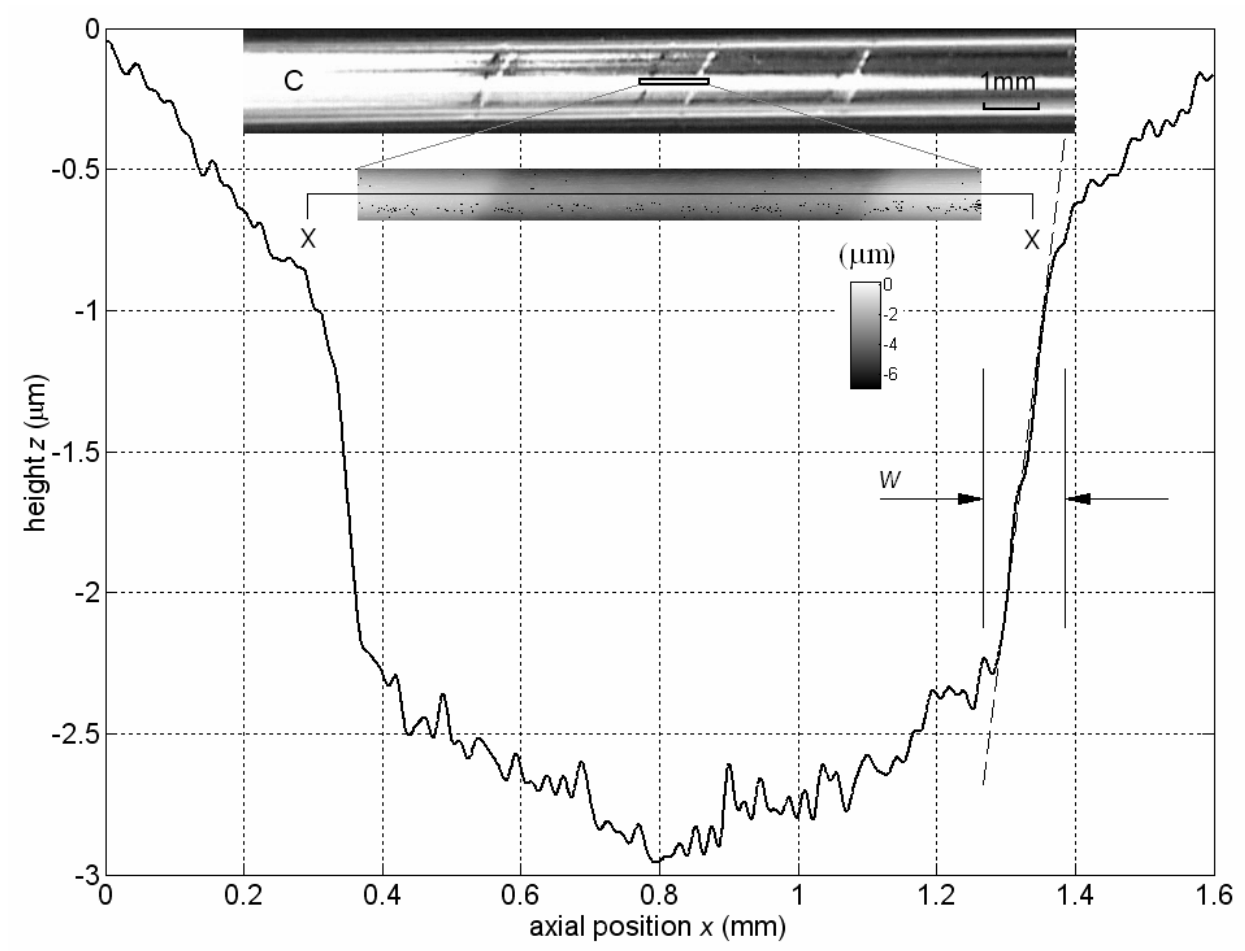

Figure 6 Surface profile of the helical domain corresponding to $C$ in Fig. 2 

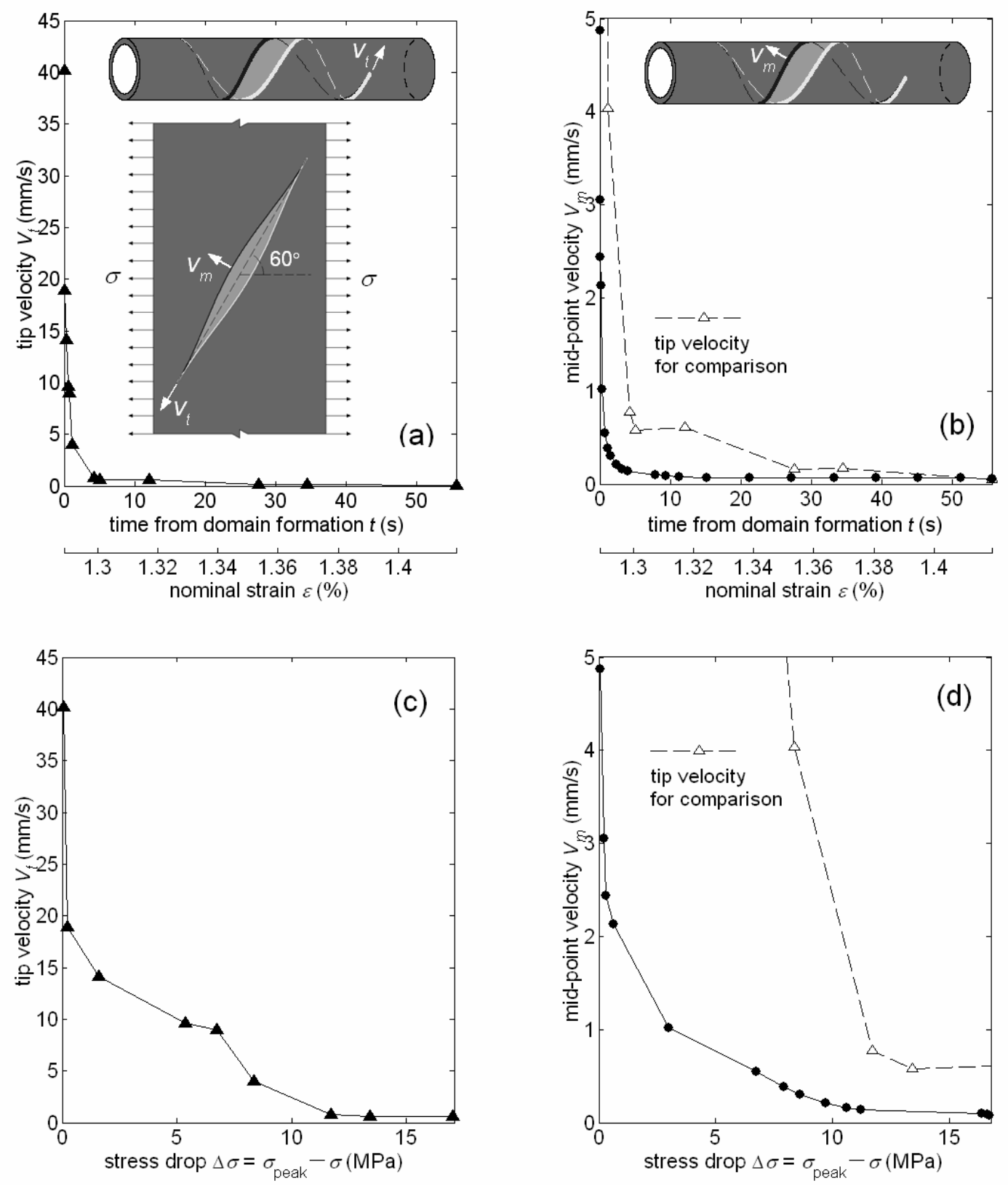

Figure 7 Front tip and mid-point velocity during domain formation: (a) the $V_{t}-t$ or $V_{t}-\varepsilon$ curve; (b) the $V_{m}-t$ or $V_{m}-\varepsilon$ curve; (c) the $V_{t}-\Delta \sigma$ curve; (d) the $V_{m}-\Delta \sigma$ curve 

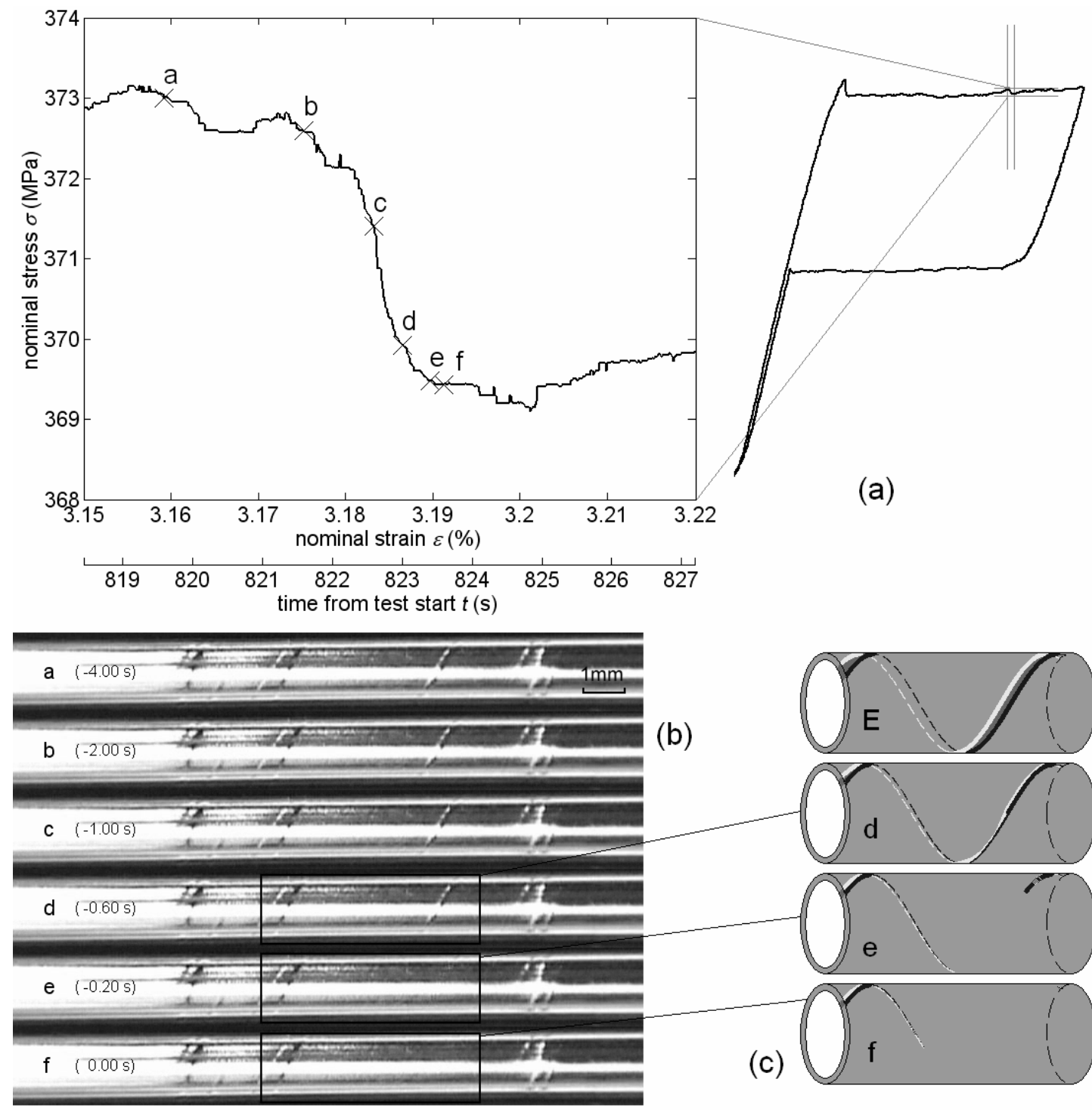

(b)
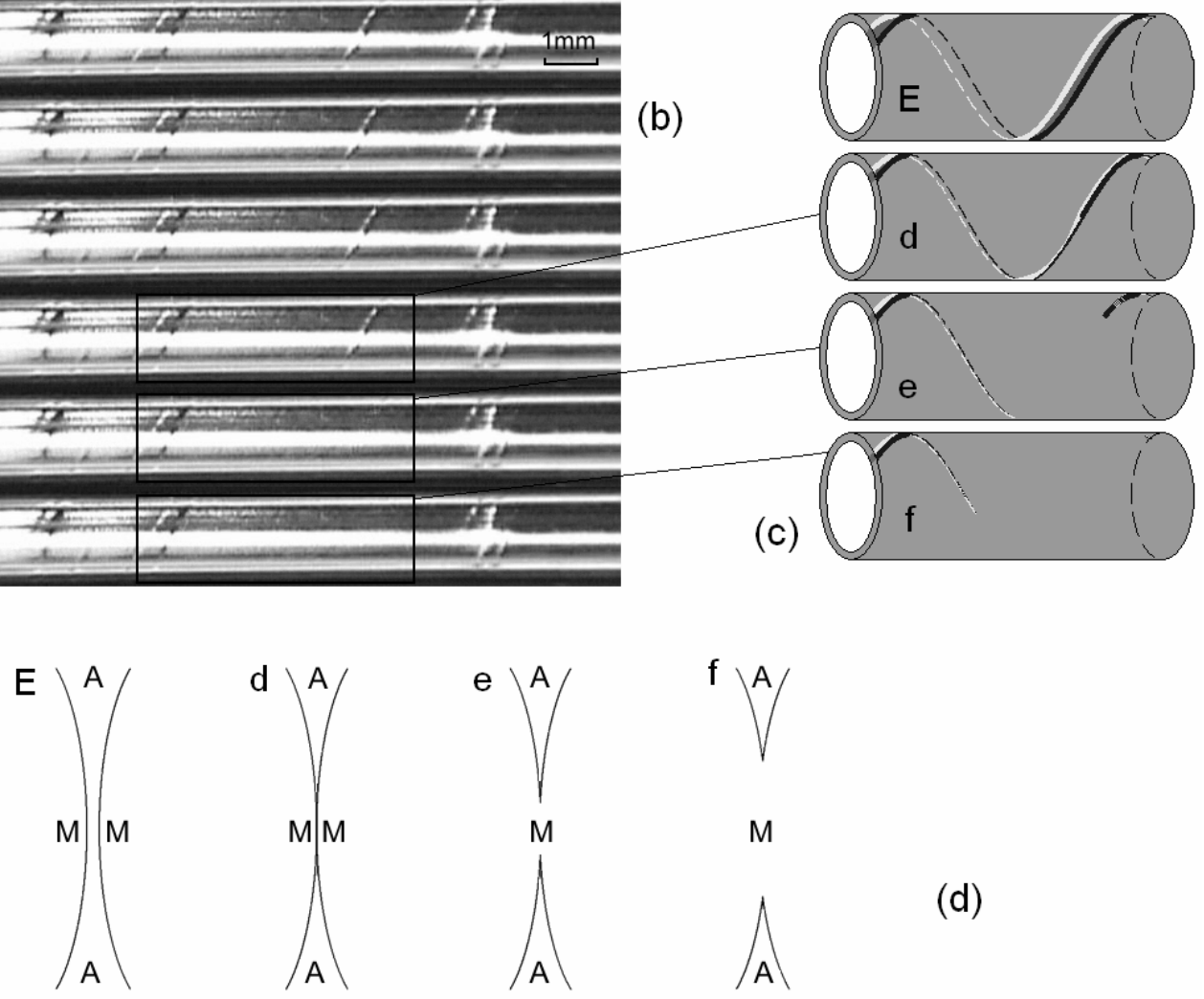

(d)

Figure 8 Self-merging of the helical domain: (a) the nominal stress-strain curve; (b) the corresponding surface morphologies; (c) 3D schematics of the morphology transition corresponding to $E$ in Fig. 2 and $d, e, f$ in (b) (light gray = high strain domain, dark gray = low strain domain); (d) unfolded plane view to illustrate the topology transition 


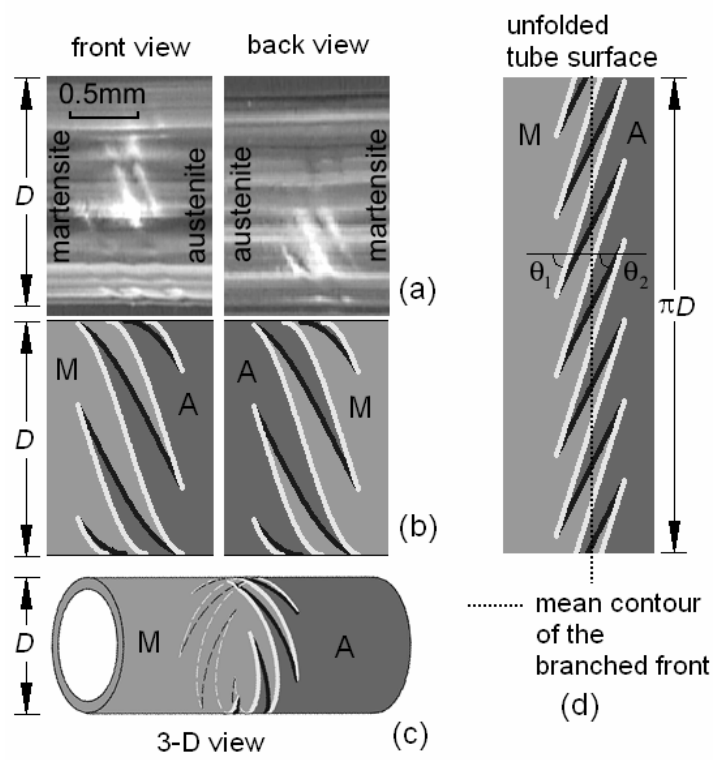

Figure 9 Geometry of the branched front: (a) front view and back view of the tube surface; (b) the contour of (a) (light gray = high strain region, dark gray = low strain region); (c) 3D view of the front; (d) unfolded plane view of the front corresponding to (b) $\left(\theta_{1}=72^{\circ}, \theta_{2}=\right.$ $\left.63^{\circ}\right)$ 

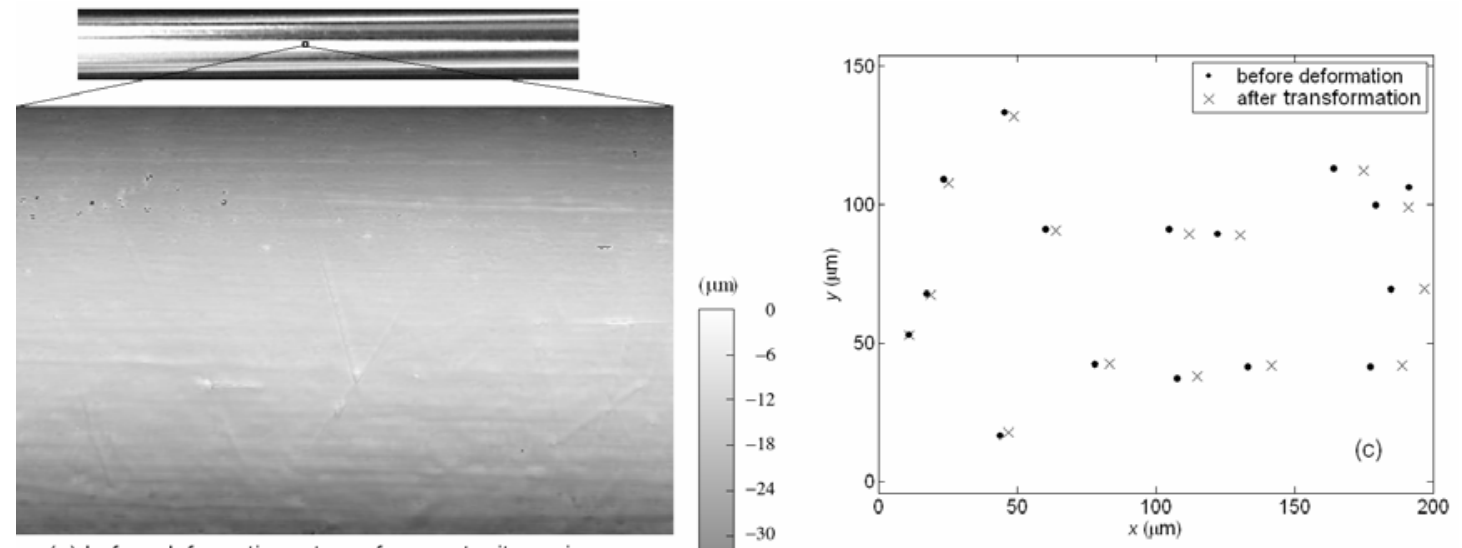

(a) before deformation: stress-free austenite region
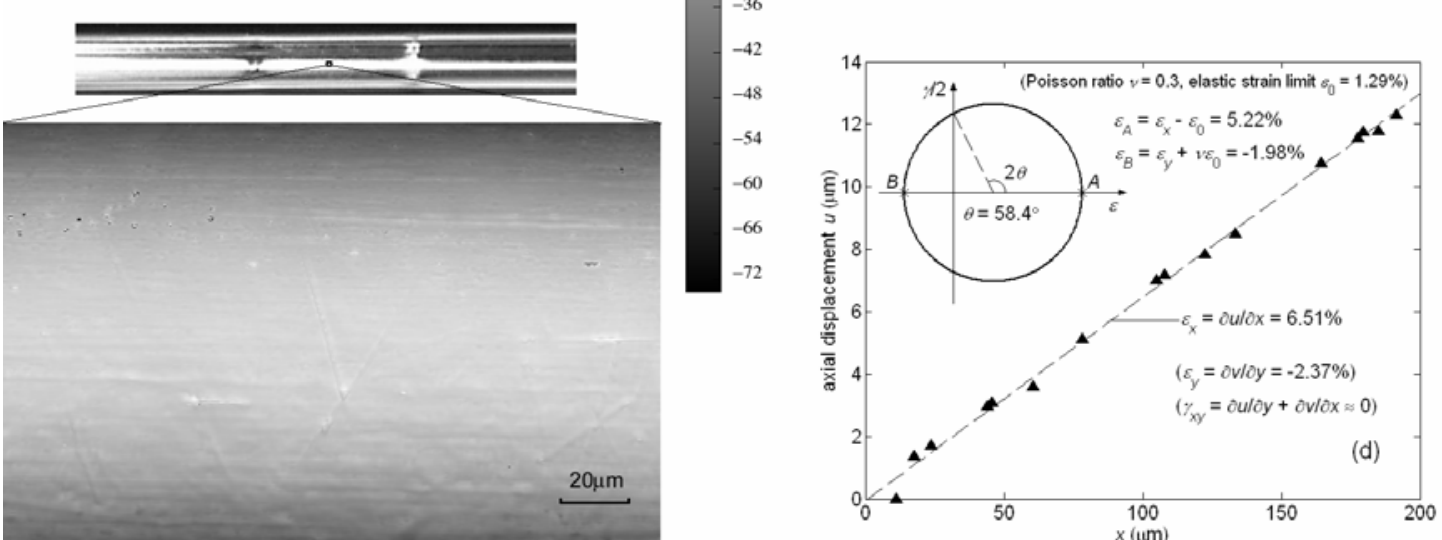

(b) after transformation: martensite within a cylindrical band

Figure 10 Surface profile of a region before and after phase transformation: (a) before deformation—-stress-free austenite region; (b) after transformation—high strain martensite region inside the domain; (c) the positions of the same particles on tube surface corresponding to (a) and (b); (d) calculation of the axial strain $\varepsilon_{x}$ and the invariant-line angle $\theta_{0}$ 


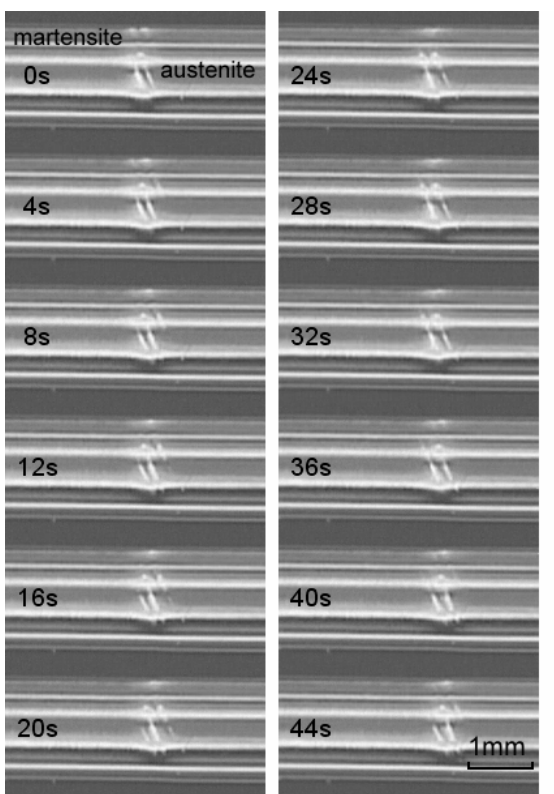

(a)

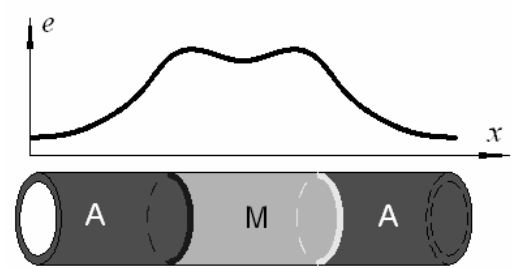

$\{$ higher strain energy

lower interface energy
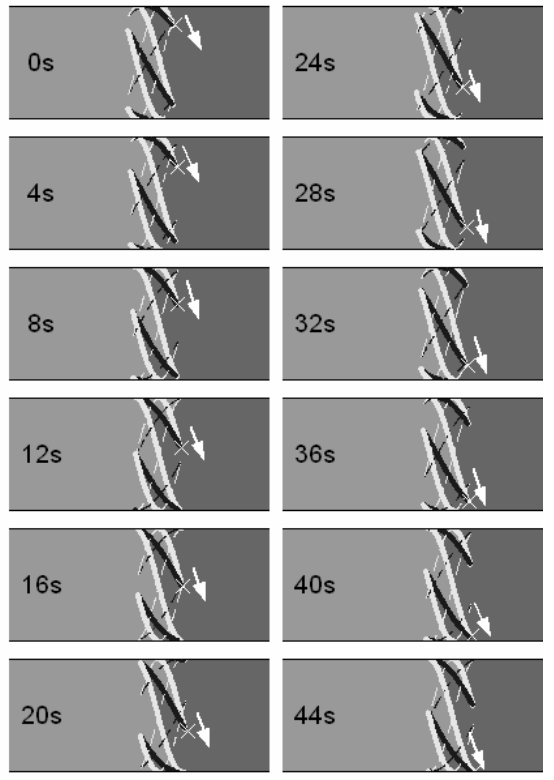

(b)

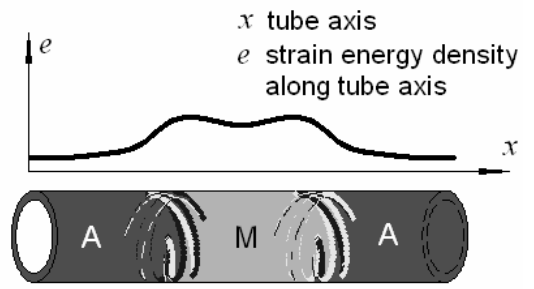

lower strain energy

\{higher interface energy

(c)

Figure 11 Convoluted motion of the domain fronts: (a) a series of photos of tube surface; (b) the contour of (a) with cross marks and arrows showing the motion of one front tip; (c) comparison of energetics between a domain with smooth perpendicular fronts and a domain of the same volume but with branched fronts (light gray = high strain region, dark gray = low strain region) 


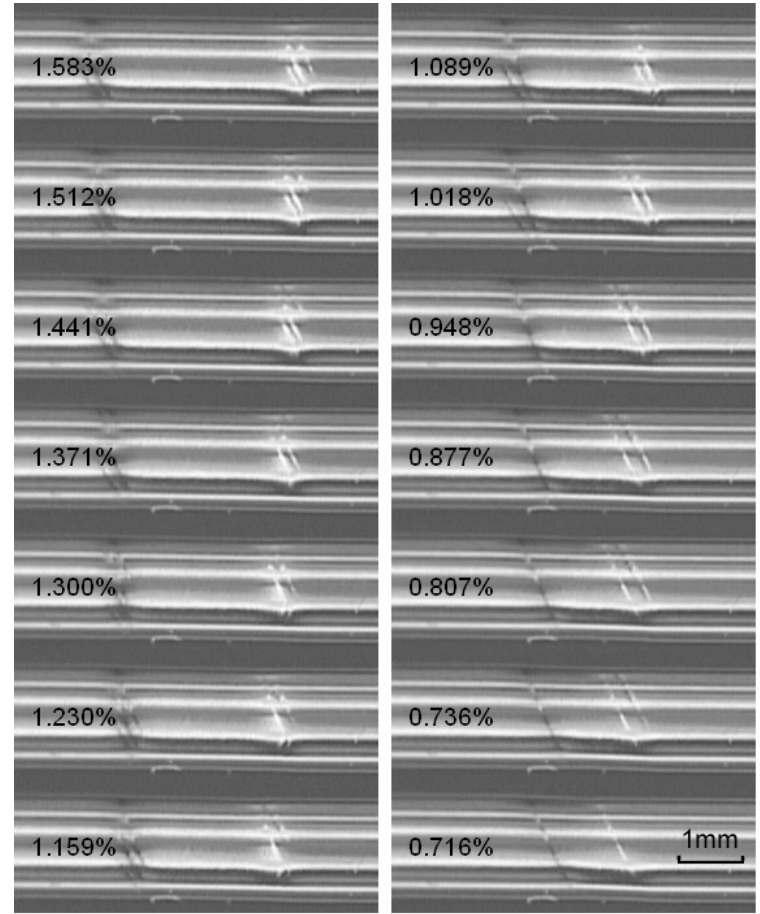

(a)

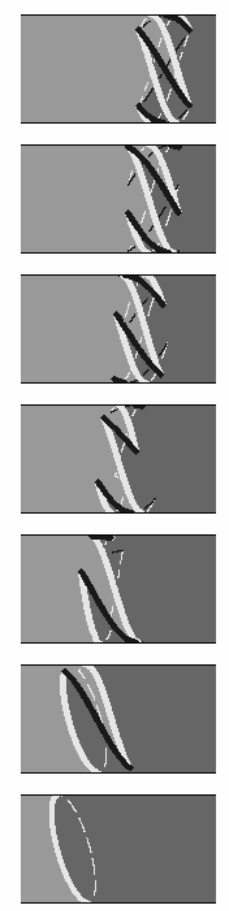

(b)
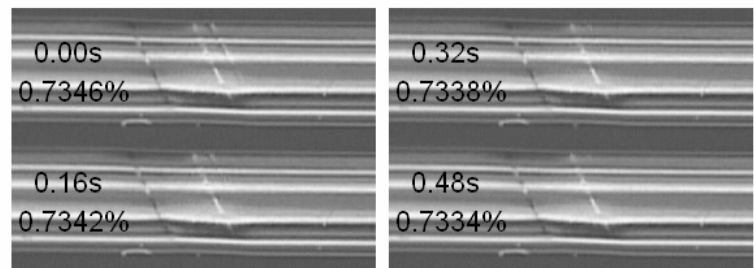

(c)

Figure 12 Unsteady morphology evolution from the cylindrical domain with branched fronts to an inclined ring with smooth fronts: (a) a series of photos of tube surface; (b) 3D schematics of the motion of the front on the right-hand side (light gray = high strain region, dark gray = low strain region); (c) photos taken during front smoothing 


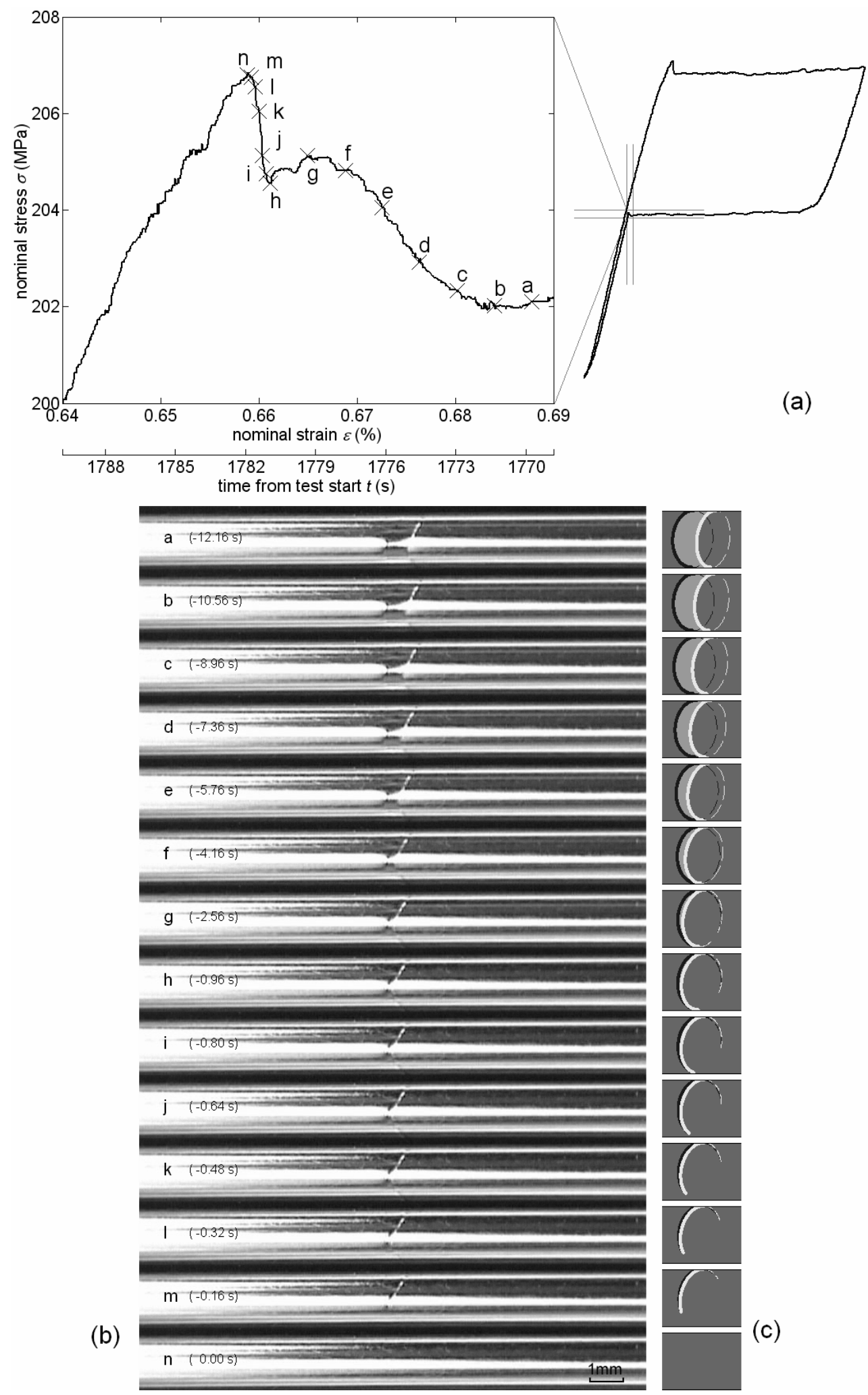

Figure 13 Ring-like domain shrinkage and domain annihilation (stage Unloading-II): (a) the nominal stress-strain curve; (b) the corresponding surface morphologies; (c) 3D schematics (light gray = high strain region, dark gray = low strain region) 

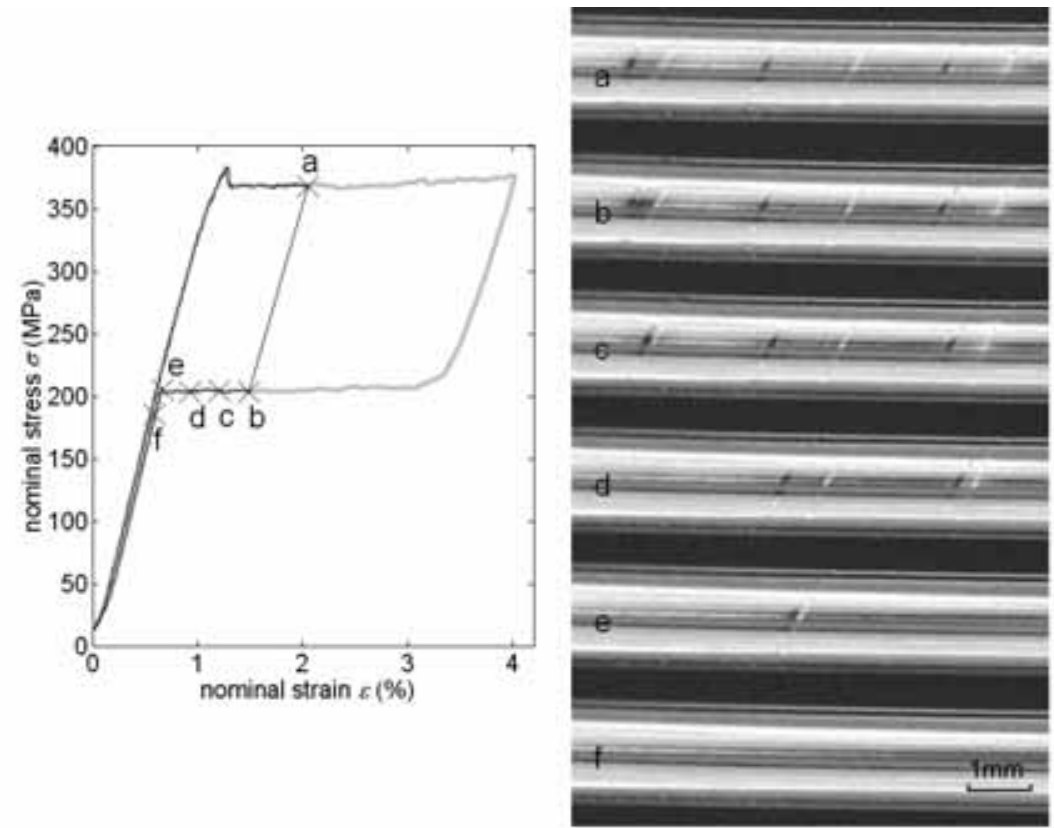

(a)
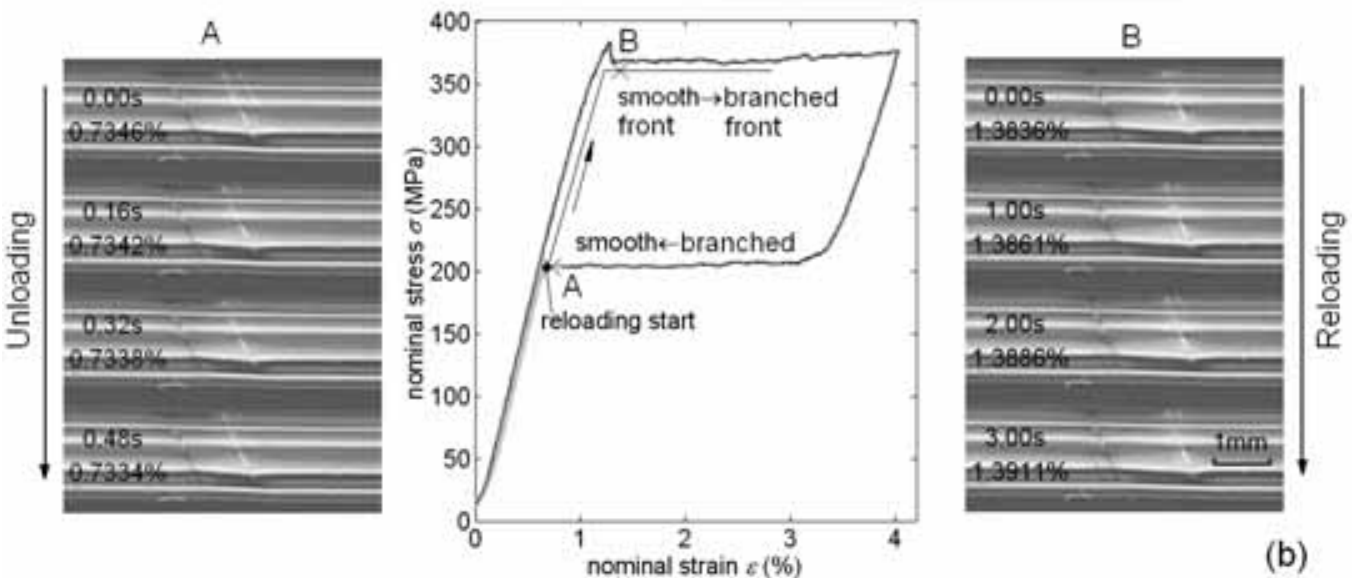

(b)
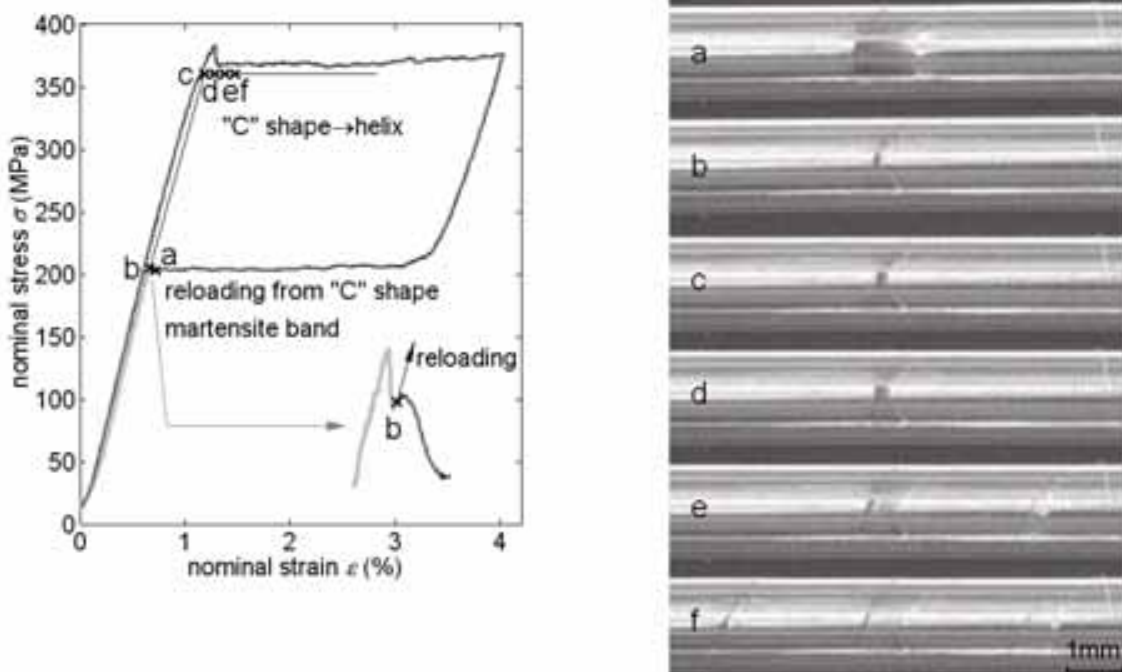

(c)

Figure 14 The stress-strain curves and corresponding surface morphologies during unloading and reloading within the loop: (a) unloading the tube with a helical domain; (b) reloading the tube with a ring-like domain; (c) reloading the tube with a C-shaped domain 

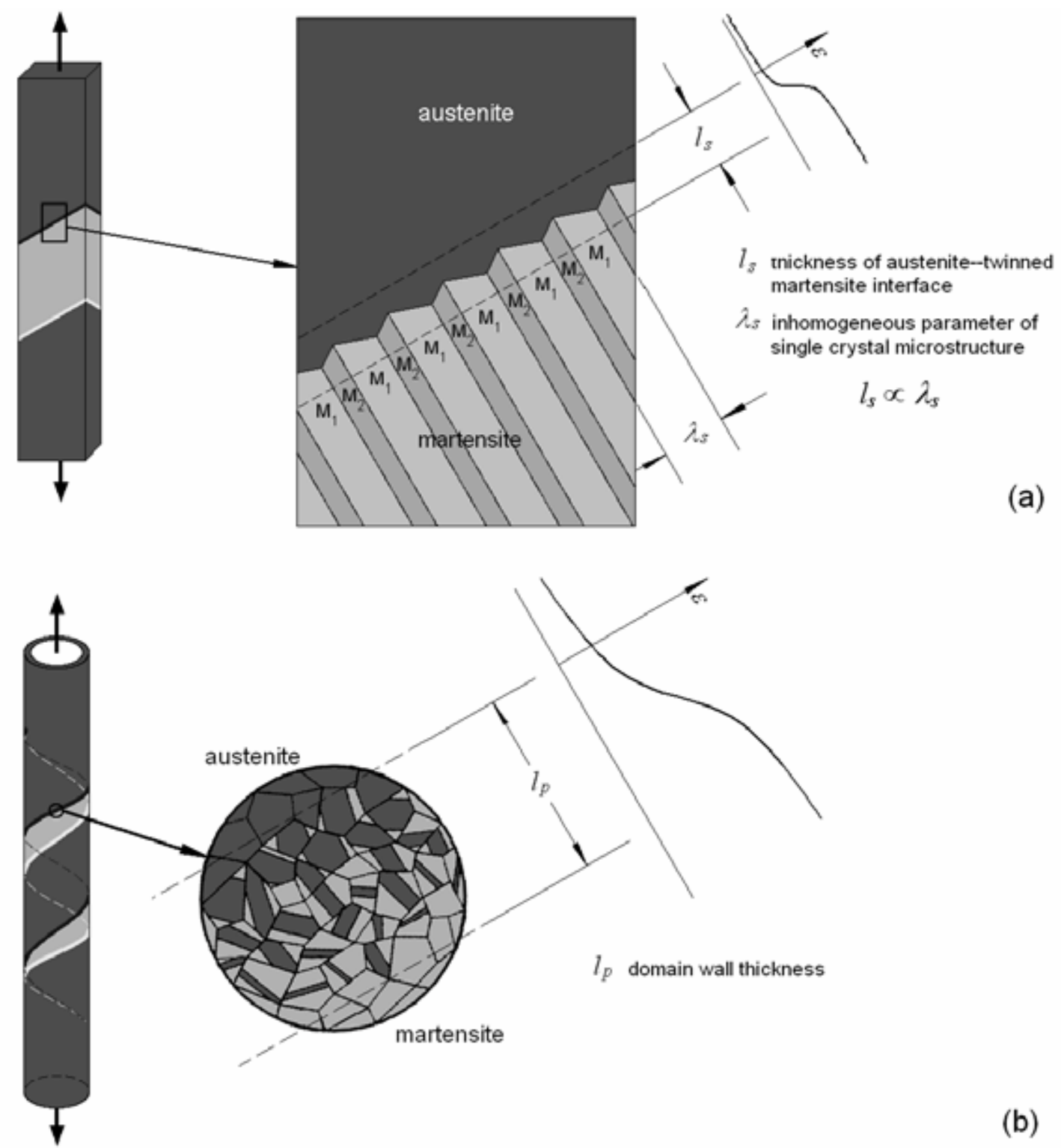

(b)

Figure 15 Multi-scale structure of the austenite-martensite interface (front zone) and the material hierarchy therein (a) interface and interface energy in single crystal; (b) domain and domain energy in polycrystal tube (light gray = high strain region, dark gray $=$ low strain region) 


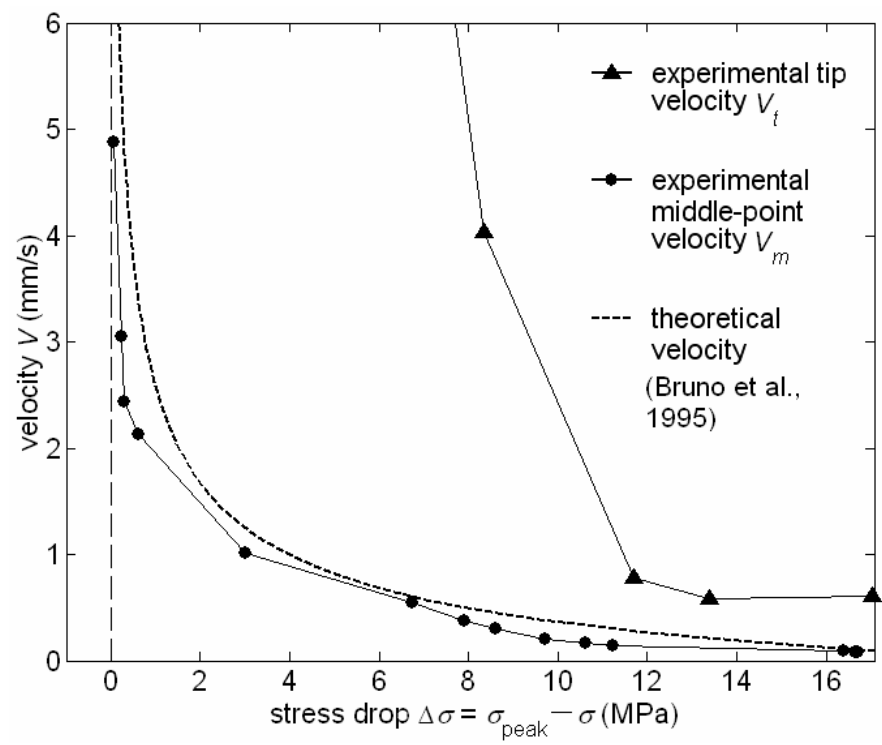

Figure 16 Variation of front velocity with stress drop 


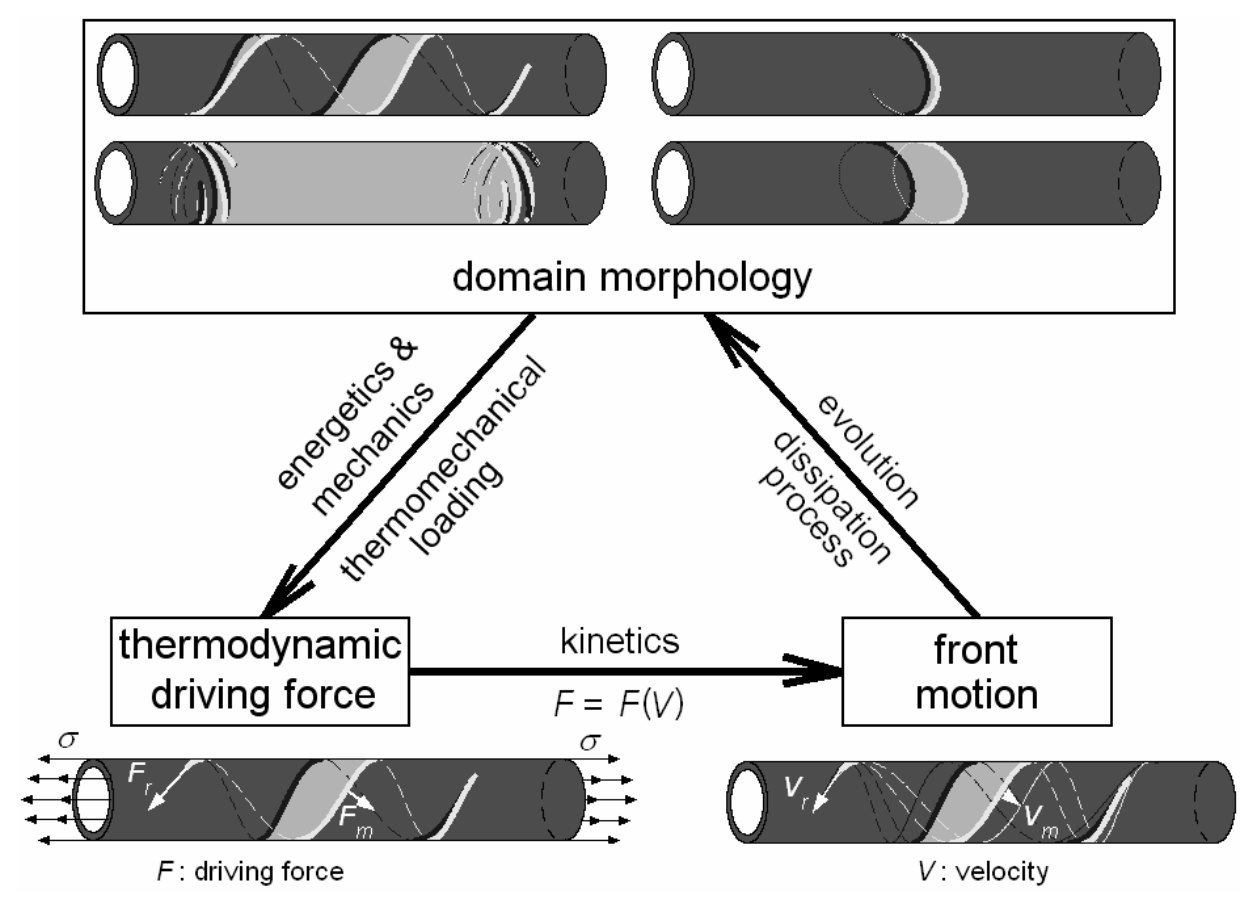

Figure 17 The interaction among domain morphology, driving force, and front motion (light gray $=$ high strain region, dark gray $=$ low strain region) 


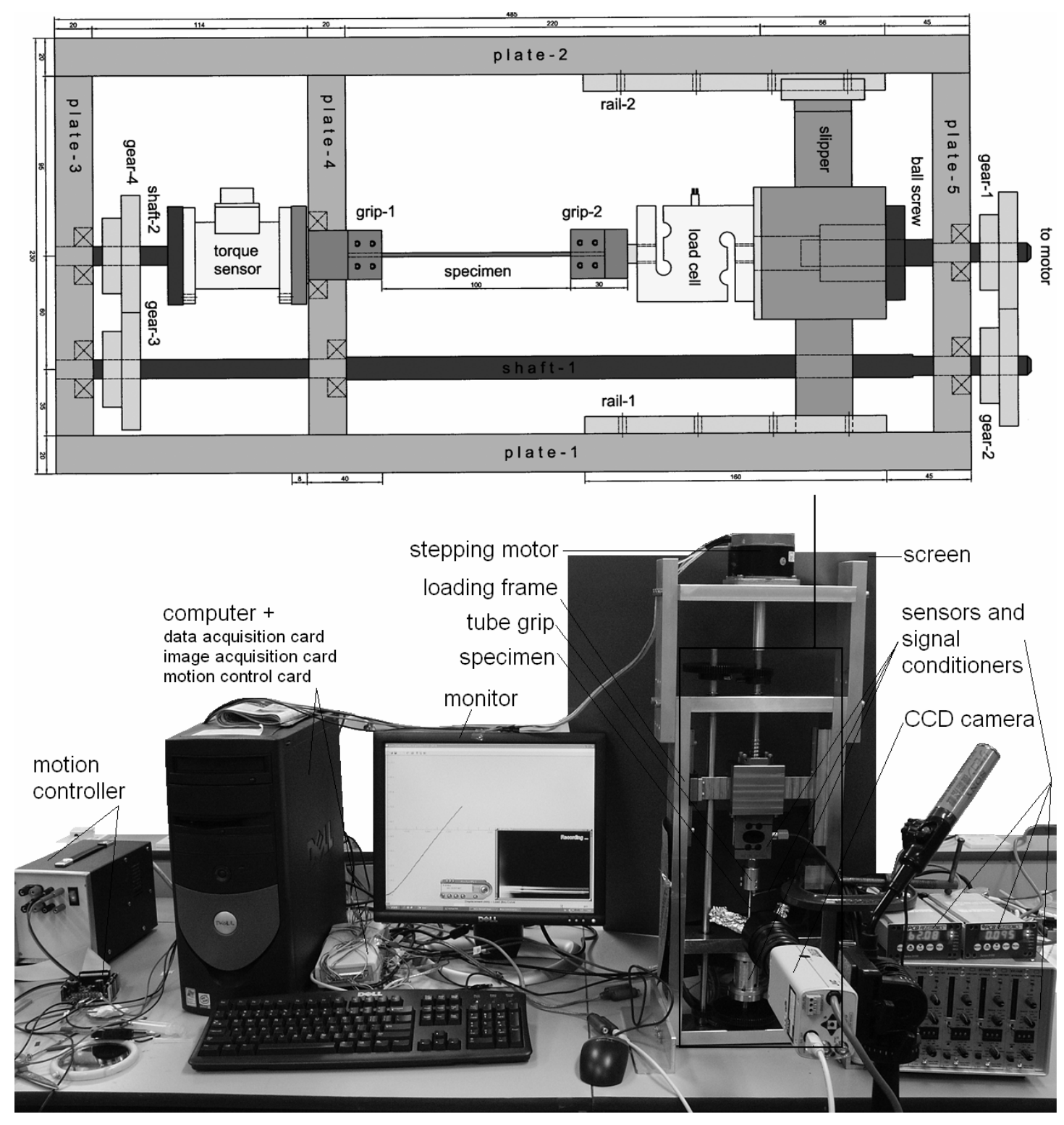

Figure A-1 Experimental setup (with the blueprint of the loading frame) 